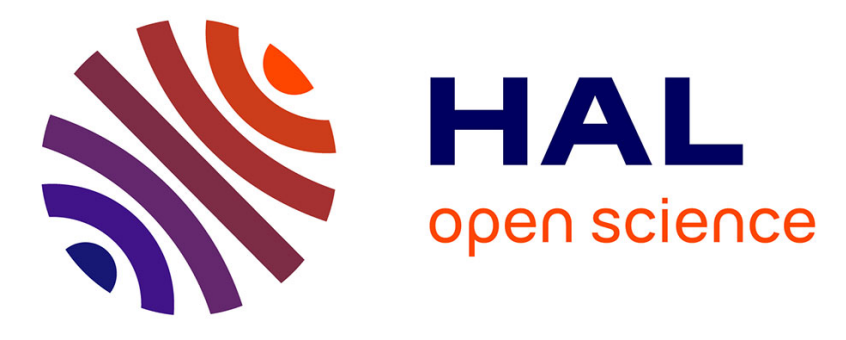

\title{
Sociétés, milieux, ressources: un nouveau paradigme pour les médiévistes
}

\author{
Joëlle Burnouf, Corinne Beck, Marie-Christine Bailly-Maître, François \\ Duceppe-Lamarre, Fabrice Guizard, Aline Durand, Carole Puig
}

\section{To cite this version:}

Joëlle Burnouf, Corinne Beck, Marie-Christine Bailly-Maître, François Duceppe-Lamarre, Fabrice Guizard, et al.. Sociétés, milieux, ressources: un nouveau paradigme pour les médiévistes. Congrès de la SHMESP, May 2007, Cergy-Pontoise, France. pp.95-132, 10.3406/shmes.2007.1947 . halshs01570448

\section{HAL Id: halshs-01570448 \\ https://shs.hal.science/halshs-01570448}

Submitted on 30 Jul 2017

HAL is a multi-disciplinary open access archive for the deposit and dissemination of scientific research documents, whether they are published or not. The documents may come from teaching and research institutions in France or abroad, or from public or private research centers.
L'archive ouverte pluridisciplinaire HAL, est destinée au dépôt et à la diffusion de documents scientifiques de niveau recherche, publiés ou non, émanant des établissements d'enseignement et de recherche français ou étrangers, des laboratoires publics ou privés. 


\title{
Sociétés, milieux, ressources : un nouveau paradigme pour les
} médiévistes

\author{
Madame Joëlle Burnouf, Madame Carole Puig, Madame Aline Durand, Monsieur \\ François Duceppe-Lamarre, Monsieur Fabrice Guizard-Duchamp, Madame Marie- \\ Christine Bailly-Maître, Madame Corinne Beck
}

\section{Citer ce document / Cite this document :}

Burnouf Joëlle, Puig Carole, Durand Aline, Duceppe-Lamarre François, Guizard-Duchamp Fabrice, Bailly-Maître MarieChristine, Beck Corinne. Sociétés, milieux, ressources : un nouveau paradigme pour les médiévistes. In: Actes des congrès de la Société des historiens médiévistes de l'enseignement supérieur public, $38^{\mathrm{e}}$ congrès, Île de France, 2007. Etre historien du Moyen Age au XXle siècle. pp. 95-132.

doi : 10.3406/shmes.2007.1947

http://www.persee.fr/doc/shmes_1261-9078_2008_act_38_1_1947

Document généré le 20/10/2015 


\section{Sociétés, milieux, ressources: un nouveau paradigme pour les médiévistes}

Joëlle Burnouf, Corinne Beck, Marie-Christine Bailly-Maître, François Duceppe-Lamarre, Fabrice Guizard-Duchamp, Aline Durand, Carole Puig

Le thème «Archéologues et historiens des textes : quelles relations? " a suscité de nombreuses propositions de communications de la part des archéologues. Nombre d'entre elles portant sur le thème des relations des sociétés avec les milieux, nous avons fait le choix de les regrouper sous la forme d'une table ronde qui permette de traiter les différentes facettes de la question posée à partir d'un objet commun, même si celui-ci reste très large et pourtant loin de couvrir le champ des possibles. Dans cette introduction, nous exposons quelques-unes des idées, largement débattues dans de nombreux colloques et tables rondes spécialisés, qui font consensus dans la «nouvelle» communauté des chercheurs qui les ont fait émerger et qui les mettent en pratique.

\section{Le poids de l'héritage}

Larchéologie médiévale est jeune : 50 ans! Depuis les fondateurs, tous spécialistes des "documents écrits" (des historiens) devenus praticiens des "documents matériels" (des archéologues), bien des choses ont changé. La question des relations entre les documents archéologiques et les documents écrits ne se pose plus. À tout le moins, elle ne devrait plus se poser si chacun y mettait du sien. Nous allons tenter de montrer pourquoi et comment.

Depuis la contribution pionnière et militante de Jean-Marie Pesez à ce débat, les conditions du " métier " ont changé, les "praticiens" se sont multipliés, mais la question de fond est encore curieusement posée ${ }^{1}$.

1. J.-M. Pesez, "Histoire de la culture matérielle ", La Nouvelle Histoire, dir. J. LE Goff, Paris, 1978, p. 191-227. 
Or il ne faut pas confondre le métier (archéologue) et l'objet d'étude (les sociétés). Historiens nous sommes, car notre objet est l'étude des sociétés. La mise en perspective choisie et assumée des documents écrits et des documents archéologiques appelle une première observation générale : nous ne sommes pas dans le même registre documentaire, car les documents des uns et des autres constituent deux niveaux de renseignements. Ce sont deux ensembles documentaires aux modes de production totalement différents, possédant leurs propres référents temporels et spatiaux. Les documents que les uns et les autres mettent en ouvre sont irréductibles entre eux, soumis à des aléas de conservation totalement différents, mais surtout soumis pour les documents archéologiques au filtre d'un travail de "déconstruction-reconstruction ": fouiller c'est détruire, ce qui exclut toute démarche de retour à la source primaire, d'interprétation et de mise en mots. L'historien sédimentaire est ainsi un " médiateur » largement juge et partie dans la " construction" des documents archéologiques qui sont sans lui "présomptifs". Il n’y a donc pas de complémentarité entre les différents types de documents.

\section{La géographicité}

des documents archéologiques

Le temps détermine l'ossature de notre objet d'étude, organise le déroulement des faits, il n'en constitue pas pour autant la structure. C'est toute la malice de Claude Lévi-Strauss, anthropologue, quand il esquive la question de la temporalité (il n'y a pas d'histoire sans date) et renvoie dos à dos l'historien, prisonnier du temps, et le géographe, dépositaire des grands espaces. Notre démarche s'inscrit davantage dans la lignée de la géographie et se rapproche de l'attitude du géographe Georges Bertrand qui tente de démêler l'écheveau des temporalités et distingue le " temps processus " du " temps référence ». L'archéologie utilise la stratification, dont l'étude est la stratigraphie. Elle en partage les méthodes avec les géographes physiciens et les géologues mais, mise en ouvre par les archéologues, la stratigraphie est rapportée à des séquences fabriquées par les sociétés ou entrecroisées avec les séquences qui intéressent géographes et géologues. Ces séquences permettent d'établir les chronologies relatives, fondées sur la succession des couches archéologiques et des artefacts et ćcofacts qu'clles contiennent, et combinées aux datations absolues des méthodes radiométriques (carbone 14) et archéométriques (la dendrochronologie). 
Depuis les " grands décapages " (vingt ans), la " fabrique du temps ", sa mesure et sa perception ont été renouvelées. La multiplication des fouilles d' "ensembles clos" (les fosses et les dépotoirs par exemple, où les objets sont en principe enfouis en même temps) a augmenté la masse des données permettant de construire de nouveaux modèles chronologiques et culturels. Désormais, "le temps s'est inscrit dans l'espace " et conduit d'une lecture verticale (stratification/stratigraphie) à une lecture horizontale, par juxtaposition. Cette dimension introduite par l'archéologie est la " géographicité " des objets d'étude : les découvertes ne prennent sens que dans le cadre de l'étude de l'espace, des réseaux et des relations aux milieux de vie et de travail, à petite échelle. Elle conduit donc à revisiter les concepts de nature, d'environnement et de paysage tels que les emploient les différentes corporations d'historiens en raison de la place des paradigmes instaurateurs dans leur définition des périodes (on pense par exemple aux topoi du type des grands défrichements du Moyen Âge ou de la déprise de la fin de la période). C'est le sens de l'interrogation sur la «nature des médiévistes ". Â grande échelle, celle de l'espace fouillé, les mêmes analyses spatiales appliquées aux lieux permettent d'interpréter autrement les fonctions et l'histoire du site.

C'est sans conteste l'irruption de ce thème d'étude sur les interactions sociétés/milieux qui a le plus transformé les interprétations des archéologues médiévistes en prenant en compte un état de l'espace et des milieux qui hérite pour partie des constructions entreprises par les sociétés à des pas de temps variables : la protohistoire pour la construction des territoires, l'Antiquité et le premier Moyen Âge pour d'autres aspects. Les résultats des études conduites sur cette question permettent de comprendre la part des hybridations, des héritages et des mobilités. Ils aboutissent à des notions majeures telles que l'anthroposystème, la mobilité de la nature à travers l'idée que les milieux ont (aussi) une histoire ou encore l'histoire des anthroposystèmes hérités.

\section{Les temporalités : la question du temps est-elle une question centrale?}

La notion de "temps processus " implique que chacun des objets étudiés, matériel, social, environnemental, dispose de sa propre temporalité et participe d'un système dont il est l'expression en un lieu donné : les temps des techniques et les temps des matériaux, les temps chronométrés, les temps de la société, les temps de l'environnement. 
L'échelle de temps commune des archéologues est exprimée en " années réelles" après calibration : les dates dites calibrées sont exprimées en «cal BP " (before present), années solaires avant 1950; "cal BC " (before Christ), années solaires avant J.-C.; et " cal AD » (Anno Domini), années solaires après J.-C. Cette échelle de temps découle pour une grande part de la compilation des datations par le radiocarbone. Elle est chronométrique et requiert une représentation sous forme de durées. Elle conserve une marge d'incertitude exprimée sous la forme d'un écart statistique de probabilité (écart de confiance exprimé à $94,5 \%$ ou $62,8 \%$ ). L'emploi de la dendrochronologie (autre méthode récente, qui date du milieu du $\mathrm{xx}^{\mathrm{e}}$ siècle) requiert une instrumentation dont la résolution à l'année, voire à la saison, offre une échelle de temps plus fine et précise mais liée à la contrainte de conservation des bois.

Mais la finesse de la résolution chronologique n'est-elle pas un leurre? En effet, il y a opposition entre un "temps référence ", précisément daté à la décennie, et un "temps processus ", celui des rythmes d'occupation en relation avec les milieux. Les enregistrements archéologiques sont représentatifs d'un " système " qui ne peut être globalisé mécaniquement. La notion de "temps processus " implique que chacun des objets étudiés, matériel, social, environnemental, dispose de sa propre temporalité et participe d'un système dont il est l'expression en un lieu donné : il n'y a donc pas "le temps" mais des temporalités.

\section{L'interdisciplinarité entre documents archéologiques et documents écrits}

Compte tenu de cette constatation, la seule pratique raisonnable permettant aux spécialistes des documents archéologiques et à ceux des documents écrits de travailler ensemble en évitant l'instrumentation, les déterminismes, la sur-interprétation ou la sous-interprétation est donc l'interdisciplinarité. Énoncer cette proposition ne résout cependant rien si on ne met pas en acte de manière explicite cette interdisciplinarité. L'interdisciplinarité ne se décrète pas, la pratique du travail interdisciplinaire des chercheurs et/ou groupes de chercheurs qui ont "intérêt " à discuter ensemble nécessite une convergence sur un même objet, une mise en jeu des forces sociales se regroupant sur un projet commun afin de construire ensemble un objet de recherche. Malgré toute la tension intellectuelle que suppose un tel projet, il constitue un élément fédérateur d'autant plus important que, "regroupés " pour ce projet, les chercheurs deviennent alors un "groupe " plus 
fort, comme cela a été constaté par les auteurs de ce texte dans leurs différents programmes : la création d'un objet commun de recherche devient alors créatrice d' "identité ". Cette construction de l'interdisciplinarité est possible et elle fonctionne bien : nos expériences le montrent depuis bientôt vingt-cinq ans.

Une fois acquise la volonté de travailler de manière interdisciplinaire, par construction d'un projet commun, vient le moment de l'intégration. Les deux mots " intégration " et " interdisciplinaire " (et non pluri-, multiou trans-disciplinaire) ont une grande importance. Le mot "interdisciplinaire" est suremployé et ne correspond malheureusement pas encore vraiment à une pratique. C'est un mot qui "rapporte", au sens social du terme, dans les réponses aux appels d'offres comme dans les commissions diverses et variées, où chercheurs et laboratoires voient leurs projets et programmes examinés. On rencontre encore multidisciplinaire et pluridisciplinaire; or les deux préfixes ne renvoient aucunement à un renversement des questionnements et pratiques mais à une simple "juxtaposition " des disciplines. Chacun arrive avec l'épistémologie et l'histoire de sa discipline, et continue à travailler comme s'il travaillait seul, en acceptant simplement - et pour certains c'est déjà beaucoup - que les résultats de ses travaux soient mis sur la table et discutés (au sens de pris en compte) dans un champ plus large.

Le corollaire de cette conception est que la mise en commun reste pratiquée "en interne", au sein d'une discipline, sans confrontation avec les autres : par exemple, les différentes disciplines des sciences de la terre vont se mettre à travailler ensemble, ou celles de la biologie végétale ou des sources écrites entre elles. C'est pourquoi l'interdisciplinarité suppose une démarche d'intégration. Cette démarche est contraignante car elle exige : premièrement, de connaître un minimum d'état de l'art des disciplines avec lesquelles on va discuter; deuxièmement, de connaître et critiquer les documents et leurs limites, et pour chacun donc d' "ouvrir ses cuisines" et de voir comment on fabrique les "sources" à partir des documents; troisièmement, de choisir un objet commun; quatrièmement, de se mettre d'accord sur un socle commun de questions à résoudre, donc de définir de nouveaux objets de recherche; et, cinquièmement, de mettre en pratique de nouvelles procédures, de nouveaux protocoles de collecte et de traitement des données. Il y a alors véritablement « renversement » de la manière de faire (de travailler) puisque les "protocoles" (et leur définition) ne sont plus en amont du processus mais en aval : quand tout le monde est d'accord sur les attendus. C'est le changement de paradigme. 
Cette intégration ramène au changement d'échelle spatiale: du vertical à l'horizontal, le "site " et l'" au-delà du site ". Elle induit surtout le choix d'une échelle spatiale pertinente pour construire l'interdisciplinarité. L'articulation entre les sources et/ou les disciplines ne peut vraiment se faire à une échelle d'étude trop vaste. C'est en s'appuyant sur de petites régions ou sur des sites-ateliers de taille réduite (comme le préconisait le PEVS du CNRS) que l'on peut le mieux évaluer le poids respectif des multiples facteurs à l'œuvre dans les phénomènes ou les événements observés, identifier les facteurs de changements et leurs interactions réciproques... Elle conduit en outre à un changement d'échelle de temps: les temporalités des documents écrits ne sont pas celles des documents archéologiques. Tant que chaque type de chercheur travaille sur la documentation qu'il maîtrise, on ose espérer qu'il connaît l'échelle de temps à laquelle cette documentation est pertinente. En revanche, l'intégration des échelles est un véritable sport de combat. En effet, il convient d'abord de comprendre que, lorsque l'on change d'échelle, on change d'objet et que les raisonnements ne peuvent donc plus être conduits de la même manière. Ensuite, il faut chercher - et c'est cela le dialogue interdisciplinaire - quel peut être le point d'accroche entre tous, chacun devant à ce stade "camper sur ses positions " et expliquer les limites des documents qu'il exploite. C'est souvent à ce moment de la discussion qu'apparaissent les " nouds de blocage " qui sont un élément fondamental du dialogue interdisciplinaire : c'est en effet lorsque le dialogue bloque que l'on touche à un véritable problème scientifique. Il faut alors travailler le nœud jusqu'à obtenir non un consensus, mais une solution.

À l'expérience, il apparaît que, dans les discussions interdisciplinaires, le plus difficile est d'amener les chercheurs à être capables de poser des questions "opératoires" dans les champs des autres chercheurs, car cela implique d'acquérir un minimum de culture des autres disciplines afin non seulement de les comprendre, mais aussi de connaître leur épistémologie (et leur habitus au sens bourdieusien), un peu comme le ferait un ethnologue. Dans le cas où il est difficile de trouver une explication, la solution est la construction de scénarios. Même chez les archéologues, où pourtant elle devrait s'imposer, cette pratique de construction des scénarios est loin de faire l'unanimité. Il s'agit, à partir des données acquises, de proposer un certain nombre d'interprétations (scénarios) probables et de dire explicitement quel est celui qui recueille pour le moment, c'est-à-dire en l'état de l'existant connu, l'approbation de tous, ce qui permet de poser le maximum d'hypothèses sans arrêter définitivement, ni fermer le champ des possibles. Outre les "nœuds de blocage", les "angles morts" sont 
pour le moins aussi intéressants. Comme leur nom l'indique, ils consistent en tout ce qui n'a pas été vu. Chez les archéologues, il est très courant d'en rencontrer (on ne fouille bien que ce que l'on connaît), de même que dans les travaux des naturalistes qui travaillent sur la période contemporaine, dans la mesure où il leur est difficile de prendre en compte la longue durée (ce n'est pas leur métier). C'est justement tout l'intérêt du dialogue interdisciplinaire que de faire émerger de nouveaux objets de recherche.

L'engagement dans l'interdisciplinarité est donc l'occasion de transferts des savoirs disciplinaires, de leur confrontation à partir de laquelle peut se construire un nouveau savoir interdisciplinaire. La complémentarité des connaissances disciplinaires s'organisant, on assiste à l'émergence de nouvelles interrogations, de nouvelles hypothèses, à la construction de nouveaux raisonnements qui, à leur tour, conduisent à de nouvelles hypothèses. Ces avancées ont permis de poser les questions des " héritages " et de leur prise en compte, et de la résilience des phénomènes à des pas de temps variables.

Pour en apporter la preuve, nous avons choisi de présenter ici quelques exemples d'études dans lesquelles la pratique de l'interdisciplinarité entre documentation archéologique et documentation écrite s'est révélée particulièrement fructueuse. Elles donneront en outre l'occasion de voir à l'œuvre d'autres formes d'interdisciplinarités, convoquées sans être débattues, comme l'interdisciplinarité au sein des sciences de la vie et de la terre ou entre les archéologues et les naturalistes. Ces études interdisciplinaires nous conduisent des vallées à la forêt, de la chasse à la biodiversité, des espaces " jardinés " aux pratiques agricoles, des matériaux exploités au sauvage et à l'inculte. Si elles sont présentées par l'un ou l'une des chercheurs impliqués dans de vastes programmes, la bibliographie montre clairement qu'il ne s'agit en rien de travaux solitaires. L'exercice, au sein de "notre " famille de médiévistes, imposait ce mode de présentation, mais cette table ronde à sept pourrait déjà faire l'objet d'une discussion interdisciplinaire entre nous! Les médiévistes sont encore très peu nombreux dans les études environnementales, mais il est indispensable qu'ils y tiennent une place. 
1. Les relations des sociétés avec les vallées (Joëlle Burnouf)

Les vallées et les basses plaines littorales sont de véritables conservatoires des archives attestant des relations croisées entre les sociétés et les milieux. Les recherches intégrées des archéologues et des géomorphologues fluviaux ont permis de démontrer le rôle majeur des sociétés du second Moyen Âge dans le forçage des systèmes naturels et leurs conséquences dans un contexte hydrologique actif au second Moyen Âge en termes de risques pour ces socićtćs. Ellcs ont mis en place un processus de vulnérabilisation des espaces dont les conséquences se font sentir aujourd'hui, un demimillénaire après les aménagements.

Dans la vallée du Rhône, à la hauteur de l'Y lyonnais, où confluent Saône et Rhône, vingt ans de recherches ont permis de faire la synthèse des observations archéologiques et géomorphologiques sur la très longue durée. Les sociétés du second Moyen Âge, installées sur les terrains rendus urbanisables par les dépôts des deux rivières, en berge rive droite de la Saône (quartier Saint-Jean), dans la presqu'île et sur la rive gauche du Rhône, poursuivent, malgré les contraintes, l'urbanisation de l'espace de confluence. Si elles prennent en compte, comme dans la presqu'île, quelques chenaux majeurs comme le "bras des Terreaux" ou l'espace de la place Bellecour, leurs aménagements contraignent durablement l'espace d'expansion des crues du fleuve.

Sur le littoral méditerranéen, dans les basses plaines du Languedoc, les petits fleuves côtiers, qui se jettent dans les lagunes en arrière d'un cordon littoral, ont tous enregistré l'histoire longue de l'action des sociétés. Le Moyen Âge apparaît comme un moment de forçage des systèmes auquel aucun réseau n'échappe. Cette fabrication/manipulation/artificialisation est à mettre en relation avec les usages agricoles des basses terres par les sociétés et l'exploitation qu'elles font de ces zones humides. Le moindre événement météorologique normal les contraignait déjà à sur-aménager, mais depuis l'abandon de ces ouvrages devenus obsolètes et la rurbanisation récente, les risques fabriqués par les sociétés médiévales se sont considérablement accrus, en raison de l'ignorance de l'histoire de ces aménagements et de leur abandon.

Dans la vallée de la Loire, les sociétés du second Moyen Âge ont entrepris la construction de digues nommées " turcies " au début du second Moyen Âge, puis "levées" à partir du Xvi ${ }^{\mathrm{e}}$ siècle. Ces digues ont corseté le grand fleuve depuis le Bec d'Allier jusqu'à Nantes. Pour les construire et les entretenir est créé, dans la seconde moitié du XIV siècle, un métier, 
« commissaire aux turcies ». La seule " turcie " archéologiquement étudiée est celle découverte à Tours, en rive gauche de la Loire, en avant du mur d'enceinte de la ville. Elle a été archéologiquement datée du XIv siècle. Elle a été quatre fois remaniée et exhaussée en un siècle. Elle était construite en terre et en sable, matériaux locaux.

Les études conduites sur ces espaces exploitent les documents sédimentaires obtenus soit par des carottages, soit par les fouilles archéologiques. Ces deux types documentaires posent un premier problème dans le dialogue interdisciplinaire: le carottage documente à petite échelle spatiale et temporelle, son exploitation requiert des analyses qui relèvent des sciences de la terre (géomorphologie, sédimentologie) et des sciences de la vie (palynologie et microfaune). Ces analyses apportent des informations qui renseignent sur différentes échelles d'espace : par exemple, la palynologie au niveau local voire régional, quand la sédimentologie peut informer à l'échelle des transferts de matériaux depuis l'amont lointain du fleuve. La documentation écrite devient exploitable à partir du XIV siècle dans les archives urbaines. Ce sont en particulier les documents comptables qui permettent de suivre les travaux sur les ouvrages construits sur, dans et à proximité du fleuve. Toutefois, il faut que les séries soient continues. Il ressort donc que la documentation écrite fournit des "dossiers " dont l'" échelle de sincérité " doit être critiquée par le chercheur. Les sources archéologiques, elles, documentent l'espace à grande échelle. Mais, si vaste que soit le décapage, il concerne un espace microscopique à l'échelle de l'histoire du fleuve. Ainsi, sur les centaines de kilomètres de digues en rive droite et en rive gauche, de Nevers à Nantes, une seule portion a été fouillée, en rive gauche de la Loire à Tours, sur une cinquantaine de mètres. Encore faut-il considérer qu'il s'agit d'un cas particulier dans la mesure où il s'agit d'une " turcie » construite en avant de l'enceinte urbaine : non seulement nous ne savons concrètement rien des digues construites en milieu rural, mais celle-ci ne correspond pas aux descriptions contemporaines qui signalent qu'elles doivent être construites (comme les ponts) avec des pieux battus en constituant la structure interne. En revanche, les effets des crues de la Loire et des embâcles et débâcles annuels permettent d'élaborer un calendrier des événements qui affectent les superstructures, des ouvrages de franchissement en particulier. Ce sont les débâcles printanières qui sont les plus destructrices et emportent une ou plusieurs piles de ponts qu'il faut reconstruire.

La tâche de dépouillement des séries est immense, elle est pourtant un préalable indispensable à toute discussion, sachant par ailleurs qu'elle requiert la participation de différentes familles de spécialistes des documents écrits 
du Moyen Âge au xIx ${ }^{\mathrm{c}}$ siècle. En effet, des premiers travaux engagés depuis quinze ans sur la Loire, il ressort que la mise en place d'un linéaire continu de digues depuis le Moyen Âge a eu des conséquences sur le fonctionnement du fleuve au xx $x^{\mathrm{e}}$ siècle. Le PAG (Petit âge glaciaire) s'achève vers 1850 . Depuis, le fleuve a connu des crues, voire des crues importantes (mais c'est le fonctionnement normal d'un fleuve), qui ont été classées en fonction de leur importance (et non en fonction de l'écart de temps) en crues décennales, centennales, millénaires. Jusqu'à la création du " programme Loire ", l'enfoncement du lit de la Loire était attribué à l'activité d'extraction des granulats dans le lit pour la construction. Les études interdisciplinaires ont permis de démontrer qu'en fait cette incision (dont l'un des effets spectaculaires a été, au milieu des années 1970, l'effondrement du pont de Tours, qui datait du XvIII ${ }^{\mathrm{e}}$ siècle) est la conséquence du corsetage du lit vif du fleuve entre deux lignes de digues. Cet enfoncement du lit correspond à ce que les géomorphologues fluviaux appellent la "variable d'ajustement ": les milieux réagissent aux aménagements et adoptent un nouveau mode de fonctionnement. Dans ce cas, la conséquence a été perceptible près de cinq cents ans après la réalisation des ouvrages. Cette résilience à cinq cents ans est observable ailleurs et pour d'autres périodes, mais elle doit encore être argumentée. L'endiguement a eu d'autres conséquences importantes : sur la qualité de l'eau, qui est plus turbide, sur la faune et sur la flore aquatiques. Une autre conséquence encore plus lourde est la déconnexion du lit vif (et lit mineur) et du lit majeur, créant un nouvel espace : le lit endigué. Cette aventure est récente et courte au regard de l'histoire du fleuve - un demi-millénaire -, elle est longue au regard de la mémoire des sociétés et requiert pour la comprendre l'intervention des spécialistes des documents écrits et des archéologues.

Pourtant ce sont les sociétés qui ont colonisé le lit majeur, se croyant à l'abri derrière les digues (il y a une ville tous les $25 \mathrm{~km}$ de Nevers à Nantes), qui sont le plus exposées aux aléas du fonctionnement du fleuve. Le risque a été construit par les sociétés médiévales et modernes, et la société actuelle l'a reçu en héritage.

\section{Relations sociétés/milieux : la biodiversité des eaux et forêts (Corinne Beck)}

Étudier les relations entre les sociétés et leur milieu est complexe et présuppose une approche ouverte, transcendant la segmentation des savoirs. Si sa caractéristique est en effet de s'inscrire dans les interfaces - interfaces 
disciplinaires, interfaces des phénomènes (durée/espace, social/biologique) -, ce type de recherche nécessite aussi et d'abord - et c'est là la première étape de la démarche interdisciplinaire - une critique interne serrée des sources mises en œuvre par chaque discipline, en l'occurrence ici de la documentation écrite si habituelle aux historiens. Les documents écrits médiévaux n'ont pas été produits dans une perspective environnementale. Est-ce à dire, alors, que les problématiques environnementales ne peuvent être appréhendées par le discours historique? Quelle est la capacité des sources écrites à aborder la question environnementale : à traduire et à articuler temporalités naturelles et dynamiques sociales, à éclairer les processus économiques, sociaux et juridiques de l'exploitation des ressources naturelles liées au monde animal par les sociétés de la fin du Moyen Âge, à révéler les conséquences, à plus ou moins long terme, de cette exploitation sur la biodiversité? Et à quel degré de connaissance des milieux la documentation écrite permet-elle d'accéder? La réponse passe par le renouvellement de la méthodologie en s'engageant dans l'interdisciplinarité des regards : renouveler le regard habituellement porté sur ces sources en en faisant une lecture au filtre du biogéographe, de l'écologue, de l'agronome ou du forestier. Ainsi en est-il des comptabilités ducales des eaux et forêts bourguignonnes de la fin du Moyen Âge.

De la dynamique des milieux, de celle plus particulière des espaces forestiers, les hommes de la fin du Moyen Âge ont eu conscience. Seulement, elle n'est pas immédiatement explicite dans les textes; elle s'exprime de manière sous-jacente et il faut aller la traquer sous les mots, surtout sous les actions que les documents relatent. La relecture conjointe des comptabilités avec des écologues et des forestiers a fait émerger de nouvelles interrogations, de nouvelles hypothèses, a conduit à la construction de protocoles d'études - comment passer de données d'ordre économique et social à des informations d'ordre biologique et écologique? -, permettant d'interroger différemment les sources écrites pour étudier les aspects environnementaux.

Cette relecture a conduit tout d'abord à préciser les attendus de la recherche même. L'origine et la finalité des sources écrites ont largement déterminé les possibilités de restituer la biodiversité des milieux forestiers et humides. Celle-ci est vue au travers de la plume d'administrateurs et de comptables ducaux. L'approche ne peut donc être envisagée qu'à partir de l'analyse des ressources ${ }^{2}$, c'est-à-dire à partir des éléments de ces milieux

2. Voir la définition donnée par P. et G. Pinchemel, La face de la Terre, Paris, 1988. 
naturels valorisés, utilisés, exploités à un degré ou à un autre - durablement ou momentanément - ou détruits par les différents acteurs de la société bourguignonne, et plus particulièrement par le pouvoir princier, décidant des espèces à protéger ou à utiliser et de celles à détruire. C'est dire qu'une partie seulement de la biodiversité des milieux forestiers et humides est abordée.

Cette démarche, pour être pertinente, nécessite également d'être attentif aux différentes échelles d'espace et de temps évoquées par les sources et à leur emboîtement. Les séries documentaires émanent de rouages couvrant des territoires fort divers : du bailliage à la châtellenie en passant par la maître foresterie, voire le massif forestier. Administration centrale ou locale, administration ordinaire ou spécialisée (la gruerie), les données émanent d'entités administratives se côtoyant, se chevauchant ou encore s'échelonnant dans le temps. Tout pareillement, divers temps se conjuguent qui sont loin d'être superposables : temps politique, temps administratif, temps de l'exploitation économique (annuel, pluriannuel, etc.) ou encore temps biologique des espèces (de quelques mois à quelques années pour les espèces animales, décennal voire centennal pour des essences d'arbres). Par cette démarche, il a été possible de repérer l'importance des divers temps de réaction de l'environnement et des sociétés, l'ampleur de leurs effets et de leur décalage dans le temps et dans l'espace, et de poser la question des héritages et des interactions entre les processus anciens rémanents et les processus contemporains.

\section{Entre héritages...}

De la biodiversité des milieux forestiers et humides bourguignons, nous ne voyons qu'un moment : deux siècles. Cette échelle temporelle, si elle a une valeur heuristique pour l'analyse sociale, peut être insuffisante pour capter les processus d'évolution des systèmes écologiques. Pourtant, les sources ducales laissent entendre que les contemporains eux-mêmes, et notamment ceux qui ont à gérer le patrimoine ducal, ont été conscients d'une évolution. Il en va ainsi des espaces forestiers. Même si le temps de la forêt n'est pas le temps des hommes, être en charge de la gestion des mêmes massifs forestiers pendant dix, quinze ou vingt ans, comme l'ont été bien des officiers de la gruerie, a conduit immanquablement ces hommes à des observations et expériences répétées, à prendre conscience de l'existence ou non de changements significatifs dans la physionomie des bois. Les modes d'exploitation et de gestion de ces espaces évoluent nettement, suggérant, par là même, une modification du couvert forestier. 
Telles qu'elles apparaissent aux XIV et $\mathrm{XV}^{e}$ siècles, les forêts ducales sont très diversement fragiles. Si on peut apercevoir une surexploitation (coupes de bois et pâturage) conduisant à une fragilisation voire à une dégradation, il serait excessif de considérer que le phénomène touche également et pareillement tous les bois : il n'y a pas une, mais des forêts inégalement touchées par les effets des activités humaines. Cette fragilisation, que les gestionnaires ducaux imputent systématiquement aux abus d'usagers, se perçoit dès la fin du XIv ${ }^{e}$ siècle. Elle se manifeste en premier lieu par l'abondance des chablis, ces arbres morts, arrachés, tombés pour cause de vent ou de tempête. Celle-ci est telle que, au $\mathrm{Xv}^{\mathrm{c}}$ siècle et plus particulièrement après 1450 , le bois de chauffage n'est plus fourni que par le ramassage de bois mort et non plus par des ventes de coupes de taillis. L'ampleur du phénomène suggère manifestement que les bois prêtent une plus forte prise aux vents, parce que les forêts ducales sont des forêts claires ne comprenant pas de sujets suffisamment âgés pour présenter un couvert plus important que celui des jeunes baliveaux pour protéger les jeunes pousses des actions éoliennes. Dans le même temps, un autre phénomène, entrevu lui aussi à travers les ventes de bois et prenant toute son ampleur au $\mathrm{Xv}^{\mathrm{e}}$ siècle, vient corroborer cette fragilité. On constate la rétraction des ventes de bois d'œuvre issu des forêts de futaie : non seulement leur nombre diminue mais ce ne sont plus que des ventes en "menu", par pied d'arbre. Est-ce pour privilégier l'exploitation d'autres ressources, cynégétiques ou pastorales, ou s'agit-il d'une mesure conservatoire afin de contrôler les prélèvements, parce que les forêts se régénèrent mal? Parallèlement, la vente de taillis aux révolutions d'exploitation fluctuantes se multiplie. Si la dynamique de ces milieux, que révèle la "relecture " des comptabilités, a été accentuée par les hommes de la fin du Moyen Âge, elle est aussi en marche depuis longtemps, héritage de pratiques plus anciennes.

\section{... et projets}

Perte de la biodiversité mais aussi enrichissement, car il faut prendre garde à ne pas raisonner uniquement sur la base de dégradation, d'érosion due aux résultats des actions humaines sur les milieux. Les sociétés ont tout autant dégradé que construit leurs milieux. Le souci de conserver et de renouveler la ressource forestière a assurément habité les hommes du Moyen Âge finissant, même s'il n’a jamais été très explicitement formulé. L'absence d'un vocabulaire adéquat, d'une formulation précise n'induit pas nécessairement la non-existence de règles de sylviculture. Les massifs boisés ducaux voient émerger en cette fin de Moyen Âge un certain nombre de règles 
d'exploitation : se mettent progressivement en place balivage, coupe « ̀̀ fleur de terre ", qui deviendra la "coupe à aire", coupe de proche en proche, etc. Sans parler véritablement de "sylviculture ", au sens où elle sera définie au XIX ${ }^{e}$ siècle, la gruerie bourguignonne, consciente de la nécessité de suivre des règles pour assurer une production ligneuse durable, met en place une autre logique d'exploitation : pour servir non plus des besoins immédiats mais une exploitation plus régulière et ordonnée, donc plus rentable aussi. Ce sont tous ces éléments, certes élaborés non sans hésitations à la fin du XIV et au XV ${ }^{e}$ siècle, qui serviront d'ingrédients de base aux forestiers du Xvir ${ }^{e}$ siècle, et à Colbert en particulier pour la constitution de la grande Réformation des forêts royales et l'Ordonnance de 1669.

\section{Le métal dans les sociétés médiévales: \\ relations sociétés/milieux (Marie-Christine Bailly-Maître)}

Le Moyen Âge correspond à un temps fort de l'activité minière et métallurgique. La période qui va du début du $\mathrm{XI}^{\mathrm{e}}$ au milieu du XIV ${ }^{e}$ siècle connaît un accroissement démographique important, une évolution des techniques agraires, architecturales et de l'artisanat, un développement du phénomène urbain, bref un accroissement des besoins et des échanges. À ce dynamisme doit répondre un dynamisme égal de la production de métal. Le fer entre dans l'outillage, la construction, l'armement, etc. Les usages du plomb et du cuivre sont considérables. L'argent est l'objet de l'attention des puissants car, du $\mathrm{VII}^{\mathrm{e}}$ au XIII ${ }^{\mathrm{e}}$ siècle, l'économie connaît un monométallisme monétaire argent avec le denier et sa subdivision, l'obole.

Pour les médiévistes, le champ de la recherche est large et prend en compte l'ensemble des phénomènes liés à l'activité minière et métallurgique : les rapports de force politiques et économiques, les incidences sur le plan social (localisation de l'habitat, évolution du statut des acteurs au fil des siècles), les flux des compétences, les grandes phases de mutations techniques (pourquoi, comment, à partir d'où, selon quel mode de diffusion), le poids des données naturelles sur le déroulement de l'activité (géologie et gîtologie, hydrologie, réserves en combustibles), l'impact de l'activité sur le paysage et l'environnement (modification de la topographie naturelle, pollution, déforestation), etc. La connaissance de cette activité hautement stratégique dépend du croisement de sources différentes. L'histoire des techniques a tout à attendre de l'archéologie et de l'archéométrie. Le Moyen Âge n'a laissé aucun traité théorique ou pratique sur le sujet. C'est au $\mathrm{XVI}^{\mathrm{e}}$ siècle que sont publiés les premiers traités, et encore ne concernent-ils 
que la "grande province germanique ". Seules une lecture croisée et une mise en perspective des données fournies par les textes, l'iconographie, l'archéologie, l'archéométrie, l'expérimentation et l'ethno-archéologie permettent d'écrire cette histoire des techniques et de la comprendre dans son contexte historique. Cette approche, globale et intégrée, amène à une relecture des sources documentaires (archives, traités), mais aussi à reconsidérer l'ensemble de la société médiévale.

\section{Les sources}

La recherche documentaire, préalable aux opérations sur le terrain et en laboratoire, se fonde sur des sources iconographiques et textuelles. Liconographie médiévale est rare dans ce domaine. Les exemples les plus fameux sont les vitraux de la cathédrale de Fribourg (1350), les vignettes illustrant le Lapidario d'Alphonse X (Castille, XIII ${ }^{\mathrm{e}}$ siècle) ou encore les scènes du graduel de Kutna Hora (Bohême, fin $\mathrm{Xv}^{e}$ siècle). Les sources textuelles livrent très peu de renseignements à caractère technique, mais informent sur l'évolution du mode d'appropriation, les fluctuations des prix des métaux, les conflits pour la possession des gisements, le mode de gestion des entreprises, etc. ${ }^{3}$.

À partir du terrain, l'historien-archéologue définit l'ampleur de l'activité et son impact environnemental; il contribue aussi à une histoire des techniques. Pour cela, il s'appuie sur la toponymie (Cros, Minière, Argentière et leurs dérivés), le contexte géologique et la topographie de surface. Haldes (amas de stériles rejetés devant les entrées de mines), fosses, effondrements signalent l'existence de "vieux travaux ". L'étude des réseaux miniers repose sur l'observation de la forme des chantiers, sur les traces d'abattage conservées en parois, sur la gestion de l'espace souterrain : exhaure, aération, circulation, éclairage. La transformation métallurgique

3. Ph. Braunstein, "Le travail minier au Moyen Âge d'après les sources réglementaires ", Le travail au Moyen Age, une approche interdisciplinaire, Louvain-la-Neuve, 1990, p. 229-338; Ph.-J. Hesse, La mine et les mineurs en France de 1300 à 1550, doct. d'État droit, univ. de Paris, 1968; M.-Chr. Bailly-Maître et S. Gauche, "Une approche du droit minier en Dauphiné (xivedébut Xv' siècle) ", La Pierre et l'Écrit, 13 (2002), p. 97-114; M.-Chr. BaIlly-Maître et M. DheNIN, "Ateliers monétaires et mines d'argent dans les Alpes ( $\mathrm{x}^{\mathrm{e}}-\mathrm{xv}^{\mathrm{e}}$ siècles). Histoire, archéologie, numismatique et archéométrie ", Archéologie médiévale, 34 (2004), p. 43-64; R. Francovich, "Per la storia della metallurgia e dell'insediamento medievale sulla costa toscana : lo scavo del villaggio minerario di San Silvestro ", Kassegna di Archeologia, 4 (1985), p. 359-382; R. Francovich, "Un villaggio di minatori e fonditori di metalli nella Toscana del medioevo : San Silvestro (Campiglia Marittima)", Archeologia Medievale, 12 (1985), p. 313-402; Mines et pouvoir au Moyen Age. Actes de la table ronde de Lyon, 15 mai 2002, dir. M.-Chr. Bailly-Maître et J.-M. Poisson, Lyon, 2007. 
des minerais laisse également des signes forts sur le terrain (amas de scories). C'est aussi dans ce champ-là que l'apport de l'archéométrie est le plus essentiel. L'analyse des déchets de métallurgie (scories, parois de four), de pièces d'architecture (Palais des papes d'Avignon, château de Vincennes), d'artéfacts trouvés en fouille (objets en alliage cuivreux) peut seule apporter des informations sur les processus opératoires ${ }^{4}$.

\section{Les relations hommes/milieux}

L'activité extractive dépend de la présence de gisements, elle est donc prédéterminée géographiquement. Ce déterminisme est particulièrement prégnant pour les minerais argentiferes généralement à l'origine d'infrastructures liées au fonctionnement de l'entreprise : aménagement du territoire, développement d'habitat, etc. La gîtologie conditionne la forme des chantiers - on n'exploite pas de la même façon un gisement stratiforme et un gisement filonien - de sorte que le milieu interfere avec l'activité humaine sur le plan technique.

Larchéologie du paysage participe à cette recherche. Les exploitations laissent des traces indélébiles : modification des topographies de surface, des réseaux hydrographiques, création d'étangs, de chemins des mineurs, etc. Les haldes sont immédiatement perceptibles (absence de végétation, reliefs anormaux par rapport à l'environnement). L'impact environnemental se mesure également à la pollution générée par l'activité. Un projet collectif de recherche, centré sur le mont Lozère, a montré que les sols d'ateliers (métallurgie du plomb argentifere, $\mathrm{XI}^{\mathrm{e}}$-XII ${ }^{\mathrm{e}}$ siècles) présentaient des niveaux de pollution extrêmes, selon les normes actuelles de l'ADEME, et que la pollution atmosphérique médiévale avait contaminé les sols dans un environnement très large ${ }^{5}$. Le poids de l'activité sur le couvert forestier est tout aussi lourd. Le bois est nécessaire aux équipements souterrains : planchers, étayages, échelles, cuvelage de puits; il est surtout charbonné pour les besoins de la métallurgie. Son exploitation intensive provoque des conflits d'intérêts, des réglementations dont les archives se

4. P. Fluzin, A. Ploquin et V. Serneels, "Archéométrie des déchets de production sidérurgique. Moyens et méthodes d'identification des différents éléments de la chaîne opératoire directe ", Gallia, 57 (2000), p. 101-121.

5. Le plomb ancien du mont Lozère (48). À la recherche des mines, des minerais, des ateliers, des paysages et des hommes (PCR dirigé par Alain Ploquin); S. BARON, Trą̧abilité et évolution d'une pollution métallurgique médiévale de plomb argentifere sur le mont Lozère, doct. géochimie de l'environnement, univ. Montpellier 2, 2005. 
font l'écho ${ }^{6}$. Palynologues, botanistes, anthracologues donnent une réalité matérielle à l'éclairage des textes : importance des prélèvements, choix des essences en fonction des besoins, restitution des forêts médiévales à partir de l'étude des charbonnières, etc.?

\section{Le métal dans les sociétés médiévales: contribution à l'histoire}

Développer chacun des points évoqués ci-dessus n'est pas possible, retenons quelques apports qui paraissent essentiels. Tout d'abord, l'histoire des techniques s'écrit sur la longue durée, rythmée par des ruptures techniques ou socio-économiques, rarement politiques. Cette recherche se fait en dehors des cloisonnements de disciplines et de chronologie classiques.

Sur le plan de l'histoire, il ne fait plus de doute, maintenant, que la possession de gisements miniers est un fait politique et stratégique, tout autant qu'économique et technique. Afin de s'assurer cette maîtrise, les puissants se sont affrontés, des spécialistes ont cherché à faire évoluer les techniques d'extraction et de transformation des minerais, et les progrès réalisés dans ces domaines ont eu des répercussions directes sur les autres secteurs d'activité : monnayage, armement, agriculture, artisanat, construction... Le cas de Largentière (Ardèche) illustre bien la rivalité qui oppose les seigneurs pour la possession des gisements argentiferes ${ }^{8}$. Les recherches conduites dans le sud de la France ont mis en évidence de véritables politiques minières menées par de grandes familles, politiques seigneuriales lentement battues en brèche par le grignotage capétien pour le contrôle des mines de métaux précieux, qui se solde par l'ordonnance de $1413^{9}$. Le cas de Pampailly, dans le Lyonnais, montre la tentative du roi de France de reprendre à son compte l'exploitation de la mine de Jacques Cour après la confiscation des biens du grand argentier, en $1450^{10}$. La naissance, au Moyen Âge, de bourgades ou de villes, dont la disparition

6. L. Caliste, Ramondens, une forêt de la fin du Moyen Âge. De la formation d'un patrimoine à son exploitation industrielle (XIIT-XV' siècles), maîtrise histoire (dir. C. Verna), univ. Paris 8, 2005.

7. Voir les travaux de Philippe Allée au sein du GEOLAB (UMR 6042, univ. de Limoges-CNRS), ainsi que Protoindustries et histoire des forêts. Actes du colloque tenu à la Maison de la Forêt (Loubières, Ariège), les 10-13 octobre 1990, dir. J.-P. MÉTALLIÉ, publié dans Les Cahiers de l'ISARD, 3 (1992).

8. M.-Chr. Bailly-Maître, "Prolégomènes à une étude des mines de Largentière en Vivarais", Mémoire d'Ardèche-Temps présent, 49 (1996), p. 3-10.

9. ID., L'argent. Du minerai au pouvoir dans la France médiévale, Paris, 2002; Fr.-A. IsamberT, Recueil général des anciennes lois françaises, Paris, 1821-1833, t. 7, p. 386.

10. P. Benoîr, La mine de Pampailly, XV-XVIIT siècles : Brussieu-Rhône, Lyon, 1997 (Documents d'archéologie en Rhône-Alpes, 14). 
- Brandes-en-Oisans à Huez - ou la survie jusqu'à nos jours - Largentière en Vivarais, l'Argentière en Embrunais, Saint-Laurent-le-Minier dans les Cévennes - sont liées à la poursuite ou l'arrêt du fonctionnement des mines, est la preuve manifeste de l'impact de cette activité sur l'occupation du sol et la structuration d'un territoire" ${ }^{11}$.

Sur le plan de l'histoire des techniques, les apports sont inédits puisque produits par l'archéologie et l'archéométrie. L'étude des réseaux miniers depuis près de trente ans a prouvé que les hommes du Moyen Âge pratiquaient l'art des mines tel que le montrent les traités du XvI ${ }^{\mathrm{e}}$ siècle. L'archéologie a également amené à reconsidérer certaines affirmations rapportées dans ces mêmes traités, comme la place de l'abattage au feu, beaucoup plus répandu que ce que l'on peut lire; malgré ses défauts, cette technique dévoreuse de bois et très polluante était généralisée, ce qui a eu une incidence directe sur la pollution et sur le couvert forestier. La collaboration avec les paléo-métallurgistes éclaire d'un jour parfois inattendu certains a priori. Ainsi le travail mené sur les tonnes de barres de fer entrant dans la construction du Palais des papes d'Avignon met en évidence que ces éléments architecturaux résultent d'une métallurgie du fer directe, alors que nous sommes en 1350, que le fer est de mauvaise qualité et provient certainement d'une multitude de petits fournisseurs : histoire des techniques et histoire d'une construction se rejoignent ${ }^{12}$.

Le mineur occupe une place de choix dans la hiérarchie des métiers au Moyen Âge. La fouille de sites comme San Silvestro (Toscane) ${ }^{13}$, Brandesen-Oisans (Isère) ${ }^{14}$ ou Saint-Laurent-le-Minier (Gard) ${ }^{15}$ témoigne d'une

11. M.-Chr. Bailly-Maître, "Les agglomérations minières au Moyen Âge en Europe occidentale ", Naissance et développement des villes minières en Europe, colloque international, Liévin-Lens, 2002, Arras, 2004, p. 215-226.

12. P. Dillmann, P. Bernardi et P. Fluzin, "Iron in Medieval Monuments. Metallographic Analysis of Irons Coming from the Palais des Papes in Avignon ", Revue d'archéométrie, 27 (2003), p. 183-192; M. L'Héritier, "L'utilisation du fer à la cathédrale de Rouen à l'époque médiévale ", Haute Normandie archéologique, 9 (2004), p. 69-78; M. L'Héritier, A. Juhin, P. Dillmann, R. Aranda et P. Benô̂T, "Utilisation des alliages ferreux dans la construction monumentale du Moyen Âge. État des lieux de l'avancée des études métallographiques et archéométriques ", Revue d'archéométrie, à paraître.

13. R. Francovich, Rocca San Silvestro, Siena, 1991; R. Francovich et R. Farinelli, "Paesaggi minerari della Toscana medievale. Casrelli e metalli ", Castrum 5. Archéologie des espaces agraires méditerranéens au Moyen Âge, éd. A. Bazzana, Madrid, 1999, p. 467-490.

14. M.-Chr. Bailly-Maître et J. Bruno-Dupraz, Brandes-en-Oisans. La mine d'argent des Dauphins (XIT-XIV siècles). Huez-Isère, Lyon, 1994 (Documents d'archéologie en Rhône-Alpes, 9).

15. M.-Chr. Bailly-Maître, "Les carreaux miniers d'une grande exploitation médiévale en Languedoc ( $\mathrm{xI}^{\mathrm{e}}$-XIV" siècles) ", Archéologie en Languedoc. Revue de la Fédération archéologique de l'Hérault, 26 (2003), p. 97-112. 
société aisée dont les acteurs disposent d'un savoir-faire recherché des dirigeants. Enfin, cette société a modelé durablement nos paysages : châteaux, systèmes hydrauliques, topographie des zones exploitées, pollution, etc. L'impact environnemental encore perceptible aujourd'hui intéresse désormais les chercheurs qui travaillent sur la modélisation de l'évolution des phénomènes sur la longue durée, comme la pollution.

\section{Des chasses forestières à la chasse aux sources : \\ le renouvellement interdisciplinaire de l'environnement (François Duceppe-Lamarre)}

"Être historien du Moyen Âge au xxI ${ }^{\mathrm{e}}$ siècle ", c'est une identité double que s'approprient d'un côté les historiens des textes, ces archéologues du savoir, et de l'autre les archéologues, ces historiens du terrain. Pour diverses raisons, les relations restent trop rares malgré de réels « objets communs de recherche ". Cependant, une tentative de rapprochement réside dans la pratique de l'interdisciplinarité, une pratique qui se trouve au cœur des interactions entre les sociétés et leurs milieux. La chasse est une interaction caractéristique du milieu seigneurial, le groupe social qui génère le plus de sources écrites au Moyen Âge et dont l'appropriation de l'environnement est majeure. Les sources écrites, chartes et comptabilités, les vestiges et l'iconographie sont donc utilisés à divers titres à partir de la question suivante : comment les problématiques environnementales, par la démarche interdisciplinaire, renouvellent-elles le discours historique à partir du thème des chasses forestières? Quatre notions peuvent guider cette démonstration : les flux, les milieux, les paysages et les territoires.

\section{Dessiner de nouvelles cartes à différentes échelles}

Le recensement et la localisation des pratiques de chasse en milieux forestiers à partir des sources écrites (principalement les chartes et la comptabilité) permettent de spatialiser ce phénomène à différentes échelles. En utilisant un système d'informations géographiques, on peut représenter une série d'informations environnementales qui contextualisent les phénomènes historiques étudiés ${ }^{16}$.

16. Par exemple l'hydrosystème, les espaces forestiers, la trame urbaine médiévale et les frontières régionales actuelles. La confrontation d'éléments contemporains et médiévaux, utilisant le procédé de la superposition de couches d'informations, démontre une pertinence d'analyse à grande échelle. 
Sans effectuer un recensement exhaustif, cette carte des attestations de chasses forestières dans le nord de la France entre le $\mathrm{XI}^{\mathrm{e}}$ et le Xvi siècle montre que tous les types de forêts sont sollicités : forêts littorales, forêts des fleuves côtiers et forêts de plaines humides de quelques centaines à plusieurs milliers d'hectares. C'est la première cartographie du genre qui allie démarches historique et géographique ${ }^{17}$. On peut aussi passer de l'échelle régionale à l'échelle locale afin d'avoir une vision plus fine des phénomènes. Les textes recensent quatre types de forêts à fonction cynégétique : les espaces boisés, les massifs forestiers, les parcs et les garennes à lapins. Ces éléments se conjuguent sur le terrain selon trois modèles. Mormal, dans la vallée de la Sambre, est un modèle centre/périphérie, qui possède un massif forestier et des espaces boisés périphériques. Mofflaines, dans la vallée de la Scarpe, est beaucoup plus petit et ne comprend que des garennes isolées sur le terrain. Finalement, Hesdin, dans la vallée de la Canche, constitue un modèle mixte ou complet, puisqu'il additionne les espaces de chasse mais aussi d'élevage des deux précédents cas en ajoutant un élément supplémentaire, un parc doté d'une muraille et d'un fossé périphérique. Dans le phénomène d'appropriation de l'espace par la noblesse, la clôture joue en effet un rôle de marqueur territorial, puisqu' elle est, d'une part, une affirmation de pouvoir qui peut être contestée, et que, d'autre part, elle concrétise une réserve d'espace dont les portes sont gardées par des agents. C'est donc un phénomène visible, c'est-à-dire un élément du paysage seigneurial.

\section{Retrouver des successions de paysages}

Or, pour retrouver cette interface, il faut lire les mentions de lieux-dits dans les archives avant de procéder à une phase de cartographie régressive qui guide ensuite des prospections de surface. Les vestiges retrouvés dans l'ancien bailliage d'Hesdin sont des fragments de mur dont les fondations et l'élévation sont inférieures à un mètre d'épaisseur, composés de moellons de craie et de rognons de silex liés par un mortier. Ce mur, dont la hauteur reste inconnue, donne une bande blanche teintée de reflets gris dans le paysage, barrière visuelle derrière laquelle s'animent les cours princières

Cette pertinence n'existe pas en revanche à petite échelle, l'échelle de résolution habituelle des historiens. Une telle cartographie est donc un outil de réflexion qui appelle une démonstration à partir de recherches d'archéologie de terrain. Ce travail d'analyse spatiale a été effectué au Laboratoire de géographie des milieux anthropisés de l'université Lille 1, laboratoire alors dirigé par Claude Kergomard.

17. La cartographie complète se trouve dans Fr. Duceppe-LAmarre, Chasse et páturage dans les forêts du nord de la France. Pour une archéologie du paysage sylvestre (XT-XVT siècles), Paris, 2006. 
successives. Les textes parlent aussi des coûts de secteurs démolis pour diverses raisons mais qui sont remplacés temporairement par des pieux de bois, puis par des travaux de maçonnerie. Ces mêmes sources financières citent également les ouvertures du parc, de multiples portes qui sont dotées de grilles.

La chronologie s'établit de deux manières. En effet, la stratification permet de recenser la succession des événements sédimentaires dont la stratigraphie illustre les principales phases de construction, d'occupation, de destruction/abandon de manière relative. La chronologie absolue est, quant à elle, donnée par les dates des chartes et de la comptabilité. Dans la réalité, de nombreux problèmes existent. Par exemple, l'origine du parc et du château d'Hesdin ne peut être déterminée par les textes, or l'absence de fouilles confine la recherche dans un cul-de-sac historique. L'emprise spatiale et ses évolutions demeurent inconnues, ce qui oblitère d'autant la compréhension des paysages qu'ont peu ou prou aménagés les dynasties de Flandre, d'Artois, de Bourgogne et du Saint Empire qui régnèrent sur ce pays.

\section{Retracer une pluralité de milieux diversement aménagés}

La richesse iconographique, grâce au mécénat princier, constitue une piste séduisante pour retracer les milieux, à défaut d'études sur les tourbières. Un tableau, dont l'original est souvent attribué à Jan van Eyck, représente une fête champêtre (musique, danse et chasse au vol) de la cour de Bourgogne localisée "dans les jardins du château d'Hesdin ${ }^{18}$ ". En effet, la comptabilité mentionne des milieux humides avec un "pavillon du marès " dans le secteur nord du parc, des fontaines, des milieux ouverts (des prés), des milieux boisés jardinés avec des pommiers, poiriers et cerisiers en plus de la pratique de la chasse au vol. Or, des oiseaux aquatiques sont également peints (cygnes, canards divers). Cependant, la plupart de ces éléments sont présents dans une kyrielle d'enluminures ou de peintures de la même époque

18. En plus des deux copies conservées, car l'original est perdu, l'une au Musée national du château de Versailles et l'autre au musée des Beaux-Arts de Dijon, voir les articles suivants : S. Jugre, "Une fête champêtre à la cour de Bourgogne ", Bulletin des musées de Dijon, 5 (1999), p. 59-69 et 76-77; J.-B. VArvre, "Chasse à l'oiseau et cour d'amour. Note sur deux tableaux de Dijon et Versailles ", Journal des savants, 1985, p. 313-339; A. VAN BUREN, "Un jardin d'amour de Philippe le Bon au parc de Hesdin. Le rôle de Van Eyck dans une commande ducale ", Revue du Louvre, 3 (1985), p. 185-192; Fr. DuCEPpe-LAMARre, "La résidence ducale d'Hesdin et sa place dans l'art curial au temps des princes des fleurs de lys (1384-1419) ", L'art à la cour de Bourgogne. Le mécénat de Philippe le Hardi et de Jean sans Peur (1364-1419), Paris, 2004, p. 160-162; ID., " Une fête champêtre à la cour de Bourgogne ", ibid., p. 162-163. 
à l'échelle de l'Europe. Qui plus est, lesdites sources financières attestent aussi de garennes à lapins, de héronnières, de cervidés, d'animaux captifs et de milieux forestiers, qui sont absents, alors qu'une singulière perspective de montagnes rocheuses se trouve ajoutée en arrière-plan du tableau! Si les sources écrites abordent peu la topographie locale, une section transversale du secteur d'Hesdin réalisée à partir des cartes topographiques actuelles recadre le débat ${ }^{19}$ : le tableau apparaît nettement comme une recomposition esthétique s'inspirant peut-être d'un événement historique dans un lieu donné.

\section{Dégager des échanges aux dynamiques complexes}

Peut-on décrire les flux d'animaux dans les réserves princières? Voici ce qui apparaît pour celle d'Hesdin après le dépouillement des comptes de bailliage conservés entre 1300 et $1315^{20}: 5747$ lapins, soit 383,1 par année, sont capturés, puis ce sont les chapons et les poules avec 1425 chapons et demi et 39 poules, soit une moyenne annuelle de 97,6 spécimens pour les volailles, arrivent ensuite les buses et les " aigles ${ }^{21}$ " (28 et 142 individus), ce qui fait 11,4 captures par an pour ces prédateurs aériens, qui sont suivis par les loutres, belettes, loups et blaireaux $(14,6,11$ et 4$)$, soit 2,3 prédateurs et omnivores terrestres tués par année de cette séquence et finalement 19 falconidés et 4 asturidés pour une moyenne de 1,6 oiseau de volière capturé (mais non tué!) chaque année entre 1300 et 1315 . Ce sont donc les animaux d'élevage (lapins et basse-cour) qui dominent très largement devant tous les autres, puisqu'ils constituent $96,93 \%$ des

19. En attendant des coupes stratigraphiques qui permettraient d'illustrer les caractéristiques microlocales de la vallée et ses évolutions.

20. L'analyse a été présentée principalement dans les deux articles qui suivent: Fr. DucepreLAMARRE, "Une génération de gestion animale au début du XIV siècle : la comptabilité du territoire d'élevage et de chasse d'Hesdin (Pas-de-Calais, France) ", La gestion démographique des animaux à travers le temps. VI' International Conference of the Association "L'Homme et l'Animal, Société de Recherche Interdisciplinaire ", Torino (Italia), 16-18 September 1998, publié dans Anthropozoologica, 31 (2000), p. 125-135, et Ibex Journal of Mountain Ecology, 5 (2000), p. 125-135; ID., "Une économie de l'imaginaire à l'œuvre. Le cas de la réserve cynégétique d'Hesdin (Artois, $\mathbf{x I I I}^{\mathrm{C}}-\mathrm{XV}^{\mathrm{e}}$ siècles) ", Les forêts d'Occident du Moyen Âge à nos jours, 24\% journées internationales d'Histoire de Flaran, dir. A. Corvol-Dessert, Toulouse, 2004, p. 39-55.

21. Les termes animaliers proviennent des sources comptables dépouillées. Ils sont uniquement retranscrits en français actuel. Hormis l'aigle, qui correspond à un rapace, sans doute du genre des balbuzards fluviatile ou pêcheur, les autres termes ne posent pas de problème d'identification zoologique. 
résultats chiffrés ${ }^{22}$. Marginaux, les prédateurs, qualifiés d'indésirables à poils et à plumes, viennent ensuite avec $2,76 \%$, ce qui ne laisse que $0,31 \%$ à la volière cynégétique! Voilà un portrait singulier fondé sur les sources financières, qui pourra être affiné à l'avenir par une critique interne et externe, c'est-à-dire en rappelant les limites de ces sources et en mobilisant, le cas échéant, des données ostéologiques.

Cette présentation à quatre volets démontre que l'écosystème des écologues à l'épreuve de la démarche des historiens de l'environnement n'est finalement qu'une construction théorique ou alors un scénario parmi d'autres. Afin de faciliter le rapprochement des pensées et des acteurs, les historiens du terrain font un premier pas vers les archéologues du savoir par leur approche interdisciplinaire de l'environnement. De plus, la mise en place de protocoles combinant différents types de sources constitue également un deuxième pas. Puisque la valse additionne trois pas, il n'y a plus qu'à attendre... le suivant!

\section{Saltus altomédiéval : entre abus de langage et réalité spatio-économique (Fabrice Guizard-Duchamp) ${ }^{23}$}

Les sources du haut Moyen Âge sont-elles trop pauvres pour que l'on puisse à partir d'elles s'interroger sur l'environnement? La carence des sources écrites est un lieu commun que l'on retrouve dans tous les prolégomènes de travaux de recherche sur cette période. Entre la modestie du chercheur et la réalité documentaire, force est de constater que le legs textuel des $v^{e}$ $\mathrm{XI}^{\mathrm{e}}$ siècles contient en effet une quantité finie d'informations. En travaillant sur les espaces du sauvage, la réalité est encore plus difficile à saisir dans la mesure où l'objet n'est pas signifié avec évidence sous la plume des auteurs. Il est donc nécessaire d'inventorier chaque mot relatif à l'environnement et d'en rechercher le sens. Il est également indispensable de se garder de transposer dans tous les lieux et à toutes les époques des catégories spatioéconomiques telles que ager, saltus et même silva, qui ont certes de grands

22. Des résultats chiffrés à partir de l'ensemble des documents conservés en archives, c'est-à-dire un fragment de la réalité historique.

23. La modestie de cette contribution à la réflexion générale de cet article ne me permet pas de développer à sa juste mesure l'ampleur des problèmes que soulève cette question. Pour les mèmes raisons, la bibliographie indiquée en notes est réduite à quelques références. 
avantages au plan didactique mais deviennent bien souvent les éléments du simple et commode triptyque d'un système défini comme agro-pastoral.

Le mot saltus est à ce titre exemplaire. Les historiens et les archéologues qui s'intéressent à l'économie rurale du haut Moyen Âge usent de cette notion empruntée à la pensée agronomique antique moins pour délimiter le champ de leur réflexion aux territoires de l'incultum ou de "nature sauvage " que pour disqualifier ces espaces réputés de faible portée économique, et ainsi concentrer toute leur attention sur les espaces de l'ager, les terres de culture, en particulier des céréales. Pourtant, chez les agronomes antiques, selon les idées qu'ils ont de l'économie rurale et de la société romaine, le saltus peut désigner un espace parfaitement intégré à une économie rurale diversifiée ou bien rejeté sur les marges peu rentables du domaine foncier, comme reliquat d'un mode de subsistance très ancien. L'arrachement au monde sauvage comme signe de l'évolution de l'humanité est en effet une idée répandue chez les Romains ${ }^{24}$. Le terme saltus, plutôt que de correspondre à un espace univoque, répond déjà à des impératifs didactiques dans le cadre d'un discours orienté sur l'économie rurale.

Ce discours n'est d'ailleurs pas repris au haut Moyen Âge. Il faut dire que paradoxalement cette société profondément rurale n'a pas produit de traité d'agriculture. Jusqu'au XII ${ }^{\mathrm{e}}$ siècle, c'est donc la littérature antique qui continue de faire autorité, bien que les lettrés du haut Moyen Âge n'y aient porté qu'un intérêt relatif, trop peu sensibles qu'ils étaient au contenu scientifique et économique, contenu dont la nature conjoncturelle, démontrée par René Martin, a peut-être rendu obsolètes ces ouvrages $^{25}$. Le mot saltus n'a pas pour autant disparu des usages, mais son sens s'est modifié. Isidore de Séville associe au saltus les espaces forestiers : "Les saltus sont les lieux vastes et silvestria (boisés, champêtres, sauvages?) où les arbres s'élèvent en hauteur ${ }^{26}$." Ce qu'il reprend dans un autre passage ${ }^{27}$. Au $\mathrm{IX}^{\mathrm{e}}$ siècle, Raban Maur parle de saltus vel silva, exprimant par là une certaine synonymie ${ }^{28}$. Du coup, le vocabulaire employé dans les diplômes

24. Cf. G. Verbeke, "L'homme et son univers de l'Antiquité classique au Moyen Âge ", L'homme et son univers au Moyen Age, éd. C. WENIN, Louvain-la-Neuve, 1986, p. 16-41.

25. J.-L. Gaulin, "Tradition et pratiques de la littérature agronomique pendant le haut Moyen Âge ", L'ambiente vegetale nell'alto medioevo, XXXVII Settimane, Spolète, 1990, p. 103-135; R. MARTIN, Recherches sur les agronomes latins et leurs conceptions économiques et sociales, Paris, 1971. 26. IsIdDORE DE SÉvilLe, Etymologiae, XIV (Patrologie latine, 1. 82, col. 523-524).

27. ID., Étymologies. Livre XVII : de l'agriculture, éd. et trad. J. ANDrÉ, Paris, 1981, p. 66-67 (\$6, 5-9).

28. Raban Maur, De universo, XIII, 5 (Patrologia Latina, t. 111, col. 367). 
et cartulaires entretient la confusion et confirme le glissement sémantique du terme saltus de l'espace inculte vers l'espace forestier. Par exemple, avant l'an mil, les textes évoquent le massif de l'Ardenne en le qualifiant 5 fois sur $47 / 48$ de saltus $(21 / 22 \text { fois de silva et } 21 \text { fois de région })^{29}$. D'autres "forêts" sont désignées comme saltus : Fiscanensis saltus, saltus Dervensis, saltus Gemmeticus, etc. ${ }^{30}$.

Au terme saltus, certains chercheurs préferent désormais celui d'incultum, dont l'usage dans les textes semble davantage correspondre à la réalité sous-jacente perçue par les hommes du Moyen Âge ${ }^{31}$. Partant de là, la question de la place de ces espaces dans l'économie rurale reste posée, et pas seulement dans leur délimitation ${ }^{32}$. L'historien des campagnes reconnaît depuis longtemps qu'il existe une interaction forte entre la part mise en culture et le reste du finage ${ }^{33}$. Au haut Moyen Âge, la question des usages de l'incultum est au centre de la vie de toutes les collectivités d'agriculteurs, et pas seulement dans le processus de réduction des terres sauvages pour augmenter la surface des terres mises en culture ${ }^{34}$.

29. Établi d'après la concordance des lemmes donnés par l'ordinateur pour tous les textes de l'« aire belge " avant l'an mil, en y ajoutant les textes écrits en dehors de l'aire pour la même période, cf. R. Nö̈L, "Moines et nature sauvage dans l'Ardenne du haut Moyen Âge ", dans Villes et campagnes au Moyen Âge. Mélanges G. Despy, éd. A. Dierkens et J.-M. Duvosquel, Liège, 1991, p. 572.

30. R. Musset, "Le nom et l'ancienne ceinture forestière du pays de Caux ", Norois, 31 (1961), p. 321-327; A. Dierkens, "La fondation et le premier siècle des monastères du Der ", Les moines du Der (673-1790), éd. P. Corbet, Langres, 2000, p. 27-44; J. Barbier, "Rois et moines en Perthois pendant le haut Moyen Âge. À propos des origines et du temporel de Montier-en-Der ", ibid., p. $45-81$.

31. Par exemple, M. Montanari, "La foresta come spazio economico e culturale ", Uomo e spazio nell'alto Medioevo, L Settimane, Spolète, 2003, t. 1, p. 301-340.

32. On trouve encore l'idée que, si la limite entre cultum et incultum n'est pas toujours évidente dans le monde méditerranéen, la frontière est, dans le Nord, fréquemment parfaitement linéaire, séparant le plus souvent champs de céréales et forêt. L'idée a été développée à l'origine notamment par A. Meitzen, Wanderungen, Anbau und Agrarrecht der Völker Europas nördlich der Alpen, t. 1, Siedelung und Agrarwesen der Westgermanen und Ostgermanen, der Kelten, Römer, Finnen und Slawen, Berlin, 1895.

33. Marc Bloch et Georges Duby, pour ne citer qu'eux, soulignaient en leur temps l'importance de l'incultum dans l'économie rurale, notamment le double visage de la forêt, à la fois inhospitalière et utile.

34. Selon un principe correspondant au développement économique de ces sociétés, comme l'affirme une longue tradition de l'histoire rurale au discours scientifique dont la genèse et l'héritage ont déjà été soulignés, cf. G. Bertrand, "Pour une histoire écologique de la France rurale », Histoire de la France rurale, éd. G. Duby et A. WAllon, Paris, 1975, t. 1, p. 37-113, ici p. 86; S. Lewuilion, "La forêt gauloise : esquisse d'un parcours littéraire, scientifique et politique de Louis de Froidour à Gaston Roupnel ", Hommes et terres du Nord, n 2-3, 1986, p. 161-166. 
Ces dernières décennies, ce sont les archéologues, notamment grâce aux progrès des études polliniques, qui ont cherché à renouveler les modèles de mise en valeur et d'exploitation des terroirs. Dans leur sillage, des historiens des textes ont entrepris de confronter les données archéologiques aux informations de la documentation écrite, participant ainsi aux recherches sur les paléopaysages, principalement dans la perspective de l'interaction avec les sociétés humaines. Le renouvellement de ces travaux montre en tout cas que ce qui relève de l'incultum est finalement un espace très humanisés ${ }^{35}$.

\section{Le complantage : approche croisée d'une pratique} agraire à partir des sources écrites, agronomiques, de l'archéologie et des disciplines environnementales (Aline Durand et Carole Puig)

En histoire du Moyen Âge, la pratique du complantage est un sujet qui n'a pas fait courir beaucoup de plumes ${ }^{36}$. Pourtant l'arbre, fruitier ou utile, est un élément organique du paysage agraire, car il est cultivé pour ses fruits ou son bois, coupé pour ses feuilles, ses branchages, géré pour ses fûts, son combustible. Mais la normalisation de la documentation écrite et les stéréotypes du vocabulaire employé dans les actes de la pratique rendent ardue la perception de cet usage. Aussi, cette pratique est-elle trop souvent ignorée. Néanmoins, les progrès de l'archéologie préventive et ceux conjoints de la

35. R. NoËL, "Pour une archéologie de la nature dans le nord de la Francia ", L'ambiente vegetale..., op. cit. n. 25, p. 763-820. C. Wiскнам, "European forests in Early Middle Ages. Landscape and land clearance ", ibid., p. 479-545; L'Homme et la nature au Moyen Age, éd. M. Colardelle, Paris, 1996; Fleuves et marais. Une histoire au croisement de la nature et de la culture, éd. J. Burnouf et P. Leveau, Paris, 2004.

36. L'aspect symbolique et culturel a été étudié : A. Grieco, "Réflexions sur l'histoire des fruits au Moyen Âge ", L'arbre. Histoire naturelle et symbolique de l'arbre, du bois et du fruit au Moyen Age, Paris, 1993 (Cahiers du Léopard d'Or, 2), p. 145-153; M. Pastoureau, "Introduction à la symbolique médiévale du bois ", ibid., p. 25-40. En histoire rurale, les références sont : M.-C. Amouretrr et G. COMET, Le livre de l'olivier, Aix-en-Provence, 1985; A. Bruneton-Governatori, Ethnographie de la châtaigne et du châtaignier, Toulouse, 1984.; R. Dion, Histoire de la vigne et du vin en France: des origines au XIX siècle, Paris, 1953; M. LACHIVER, Vins, vignes et vignerons: histoire du vignoble français, Paris, 1988 ; P. Leveau, C. Heinz, H. Laval, P. Marinval et J. Médus, "Les origines de l'oléiculture en Gaule du Sud. Données historiques, archéologiques et botaniques ", Revue d'archéométrie, 15 (1991), p. 83-94; P. TOUBERT, Les structures du Latium médiéval. Le Latium méridional et la Sabine du IX à la fin du XIF siècle, Rome, 1973. Ce n'est que récemment que la fructiculture a fait l'objet d'approches globales, voir le dossier spécial «Fructiculture », coordonné par M.-P. RuAs, dans Archéologie du Midi médiéval, 23-24 (2005-2006), p. 4-206. 
bio-archéologie et du paléo-environnement conduisent à la redécouvrir. En effet, le croisement disciplinaire avec les données textuelles éclaire l'histoire rurale sous un angle neuf, en restituant les techniques agraires et les formes du paysage. Le complant est une pratique qui consiste à associer des plantations arboricoles à une autre culture (céréales, vigne, etc.), mais qui peut s'étendre à toute forme d'association culturale. La définition même induit un paysage à l'architecture particulière. La société médiévale méridionale a-t-elle bâti un tel paysage? Si oui, quelle en est l'importance?

La relecture plus attentive des actes du quotidien restitue à l'arbre fruitier et au complantage une place longtemps considérée comme marginale. Les cartulaires, en effet, ne le placent pas au premier plan. Les signes identifiant cette pratique sont ténus : ce sont les vinea cum arboribus et les corvées de ramassage d'olives intégrées dans le cycle des travaux viticoles ${ }^{37}$. Les traités d'agriculture font écho à la documentation issue du quotidien : ils contribuent à valider l'existence et l'usage de cette technique, même s'ils ne correspondent pas à la même aire géographique ou à la même période chronologique. Chez Pierre de Crescent ${ }^{38}$, la pratique du complantage n'est pas non plus clairement identifiée comme telle : ainsi, au Livre IV entièrement consacré à la vigne, il observe que, dans un grand nombre de régions de l'Italie, la plantation de vigne se fait sur les berges des fossés et dans les champs près de grands arbres. Mais c'est au chapitre où il traite du moment et de la manière de planter la vigne qu'il est le plus explicite sur le sujet, bien qu'il n'y emploie pas le terme complantatio. D'abord en fournissant une liste d'essences complantées au vignoble: orme, opulus, saule, peuplier, frêne, cerisier, noyer, prunier et semblables; en zone humide, il conseille plutôt un complant avec du saule et de l'orme. Il donne un espacement précis entre la fosse de vigne et celle d'arbre : si l'arbre est trop près, il empêche la croissance de la vigne ou bien est opprimé par elle. Plus avant dans le chapitre, il fait état de cultures mariées arbres et vigne en édictant des recommandations pour le semis et la taille. Au total, les textes décrivent plusieurs formes de complant : une simple juxtaposition

37. Les complants languedociens les plus anciens sont dans le cartulaire d'Aniane, p. $431, \mathrm{n}^{\circ} 313$ (829), et p. 433, $n^{\circ} 314$ (978), ainsi que dans celui de Nîmes, p. 149, no 93 (996). On trouve des corvées de ramassage d'olives dans un cycle de travaux viticoles dans le cartulaire de Gellone, p. 398, $\mathrm{n}^{\circ} 490$ (1131). En Catalogne, la pratique est attestée dès le haut Moyen Âge : J. M. SALrach, « La vinya i els viticultors a la Catalunya de l'alta Edat Mitjana", Colloqui d'historia agraria, vinyes $i$ vins: Mil anys d'historia, Actes i communicacions del III colloqui d'historia agraria, Barcelone, 1996, p. 117-145.

38. Pier de Crescenzi, Ruralia commoda. Das Wissen des vollkommenen Landwirts um 1300, éd. W. Richter, Heidelberg, 1996. 
d'arbres dans le même champ, des arbres servant de soutiens vifs, des sujets se trouvant à la périphérie de la parcelle. L'un des problèmes soulevés par les textes savants, plus fermes et précis en la matière, est de savoir si ces recommandations sont suivies d'effet.

C'est à l'archéologie du champ, née du décapage de grandes surfaces dans le cadre de l'archéologie préventive, que l'on doit d'en avoir apporté les preuves et conduit à cette réhabilitation textuelle d'une pratique agraire. En ouvrant la voie à l'étude des vestiges agraires, cette nouvelle branche de l'archéologie change la définition même du site archéologique et fait des terroirs anciens des objets archéologiques à part entière. Initiée par les antiquisants, l'archéologie agraire est désormais prise en compte par les médiévistes et les modernistes. Sur le présent sujet, la fréquence des découvertes dans le sud de la France permet d'avancer des proportions intéressantes. En Roussillon, sur cinq opérations archéologiques qui ont révélé des plantations médiévales, deux portent des traces de complant vigne/arbres ${ }^{39}$. En Languedoc, sur trois vignobles découverts, un seul porte des traces de complantage. Ce qui fait, pour l'heure, une moyenne d'une vigne complantée sur trois dans les zones de plaine littorale. Les découvertes de vignobles antiques, en revanche, témoignent peu de cette pratique qui apparaît donc comme une particularité médiévale. Ces données sont confortées par les sources textuelles qui indiquent des arbres dans tous les types de parcelles, mais surtout dans les vignes ${ }^{40}$. Elles nous signalent qu'il existe d'autres formes de complant, associant la vigne à des plantations horticoles (céréales, légumes, légumineuses), comme c'est le cas dans le cartulaire d'Aniane (XII ${ }^{\mathrm{e}}$ siècle) ou encore les coutumes de Perpignan (milieu du XIII ${ }^{\mathrm{e}}$ siècle) ${ }^{41}$. L'archéologie agraire informe également sur les taux d'encépagement pour la vigne, mais aussi, dans le cas du complant, sur les fréquences de plantation arbustive. L'exemple d'Orle-Ouest agraire a démontré une densité de plantation arboricole équivalente au tiers et au sixième d'une éminée

39. H. Peritot, La déviation de la RD 22c, Perpignan-Cabestany, DFS, SRA Languedoc-Roussillon, Montpellier, 1998.

40. Dans les clos, comme l'atteste le cartulaire de Nîmes, p. 159, n 101 (1006), p. 177, n 112 (v. 1015); mentions les plus anciennes dans le cartulaire de Nîmes, p. 322, n 203 (v. 1114), et aux Arch. départ. du Gard, H 665 (1114). En Roussillon, les arbres sont mentionnés dans des jardins (Perpignan, médiathèque, J.-B. AlarT, Cartulaire roussillonnais manuscrit, t. 11, p. 425, Arch. de l'hôpital Saint-Jean, liasse 23, $\mathrm{n}^{\mathrm{n}}$ 50), des vignes (ibid., t. 10, p. 581, Arch. de l'hốpital Saint-Jean, liasse $31, n^{\circ} 28$ ), des terres et des champs (ibid., t. 9, p. 457; t. 100, p. 14) et des ferragines (ibid., t. 11, p. 438 ).

41. Les coutumes de Perpignan, Nîmes, 2003. 
roussillonnaise ${ }^{42}$. Enfin, les disciplines bio-archéologiques et paléo-environnementales soulignent la place de l'arbre, particulièrement de l'olivier, dans le paysage agraire, complétant ainsi l'apport de la documentation écrite.

Le croisement des données textuelles, archéologiques et paléo-environnementales éclaire l'histoire rurale dans ses techniques et ses modes d'exploitation. Cette démarche permet également de redessiner les formes du paysage. Elle lève le voile sur une agriculture " parallèle ", difficilement perceptible dans les actes de la pratique, mais qui participe à l'économie et aux modes de consommation de la société médiévale.

\section{Conclusions}

L'émergence de cette thématique a changé d'abord les pratiques scientifiques : celles du dialogue interdisciplinaire entre les spécialistes des sciences de la terre et de la vie et ceux des sciences de l'homme et de la société. L'intégration des uns et des autres au sein d'équipes de terrain et de laboratoires, la confrontation des résultats et l'interprétation intégrée des données ont donné une impulsion décisive à ces travaux. Plus que la confrontation des résultats qui est un dénominateur minimum de pratique scientifique commune, c'est la façon d'interroger les données, qu'elles soient écrites ou sédimentées, qui a fondamentalement changé, notamment par la recherche et la multiplication d'indicateurs "socio-naturels" pour comprendre les faits, obligeant le chercheur à une relecture de ses propres sources à l'aune d'autres regards nourris (géographiques, agronomiques, etc.). Cela a donc une répercussion à la fois en amont des opérations, au niveau des choix "scientifiques", et en aval, par la multiplication des possibles regards et analyses ultérieures générées par ces opérations, souvent sur des temps très longs : les analyses se poursuivant bien après la rédaction des rapports ou la publication des résultats bruts.

\section{La position des chercheurs}

De l'émergence de ce champ, de la mise au point de protocoles spécifiques, de la pratique de l'interdisciplinarité sont nés des chercheurs " hybrides ", au sens où les définit Bruno Latour. Aujourd'hui, les historiens s'interrogent

42. J. Kotarba, A. Bergeret, D. Peyruc et C. Puig, RN9, Contournement du Grand Saint-Charles, Perpignan, Orle-Ouest agraire, DFS, SRA Languedoc-Roussillon, Montpellier, 2000. 
mieux sur les textes : donnent-ils à voir le milieu ou seulement l'écriture sur le milieu, une écriture qui crée l'illusion d'un immobilisme par la répétition de lieux communs dans les sources narratives notamment? Avant de vouloir interpréter, comprendre, expliquer, il faut commencer par se mettre dans les conditions de décrire, donc s'affranchir de l'impression de "déjà-vu " et raisonner en termes de "contiguïté spatiale", c'est-à-dire aller vers une "présentation synoptique " des descriptions pour relativiser le point de vue synchronique et systémique. Ce qui a changé est aussi l'intégration de différentes échelles de temps: une échelle de temps courte, au regard des systèmes bio-géo-physiques, mais longue au regard des changements socio-spatiaux, ou inversement parfois (par exemple, lorsqu'on cherche à mettre en évidence des fluctuations environnementales). Dans le premier cas, cela a permis de mettre en évidence des seuils d'irréversibilité dans la co-évolution des systèmes et aussi les pratiques de forçage des systèmes dont les sociétés actuelles héritent directement. Ce sont aussi les rythmes des processus eux-mêmes qui sont accessibles. L'émergence de cette thématique a permis également de varier les niveaux d'analyse (macroet micro-histoire) et de les emboîter à diverses échelles spatiales.

Parmi les résultats majeurs de ces nouvelles recherches, on peut retenir ceux acquis sur les relations entre les sociétés et les milieux de vallée, les sociétés et les sols et l'archéologie agraire, les sociétés et la biodiversité, la promotion du sédiment comme objet archéologique à part entière, ainsi que la chaîne opératoire du traitement des matériaux et des ressources. Le sédiment conserve les "invisibles de l'histoire": toutes sortes d'écofacts qui renseignent autant, sinon plus pour les "structures discrètes", sur les pratiques des sociétés que sur l'activité biologique au cours du temps. En effet, pendant longtemps l'archéologie s'est intéressée aux structures mises au jour, délaissant l'" emballage ", la terre, malheureusement dénommée "remblai ». Or, ce sol enregistre finement tout ce qui arrive et documente les pratiques des sociétés.

La grande "révolution" de ces vingt dernières années est d'avoir démontré la capacité des sources écrites à aborder la question environnementale pour peu qu'on porte sur elles un regard neuf : une lecture par le filtre du biogéographe, de l'écologue, de l'agronome ou du forestier (ainsi pour les comptabilités des eaux et forêts bourguignonnes de la fin du Moyen Âge). Autant de nouvelles approches qui ont conduit à la construction des protocoles d'études permettant de mieux interroger les sources écrites pour étudier ces questions, qu'il s'agisse de biodiversité animale et végétale, de relations sociétés/milieux (de vallées, de montagne, des zones humides) mais aussi de relations sociétés/ressources : les travaux introduisent 
à des réflexions sur les forçages, les risques, les pollutions, etc. L'autre acquis fondamental est l'abandon des traditionnels clivages entre approche naturaliste, d'une part, et approche sociale, d'autre part, et l'émergence de l'approche systémique qui met l'accent sur l'imbrication et la succession - dans le temps et dans l'espace - des phénomènes à l'œuvre, des diverses causalités naturelles et sociales en jeu dans les interactions entre les sociétés et leurs milieux.

Dans cette perspective, il faut aussi insister sur l'importance de la diversité des temps de réaction de l'environnement et des sociétés, de l'ampleur de leurs effets et de leur décalage dans le temps et dans l'espace, d'où la mise en évidence des héritages. Ces études ont montré l'importance des interactions entre les processus anciens rémanents et les processus contemporains : telles qu'elles apparaissent aux $\mathrm{XIV}^{e}$ et $\mathrm{XV}^{e}$ siècles, les forêts ducales bourguignonnes résultent sans doute bien plus de la résilience des pratiques anciennes que d'une pression contemporaine. La fragilisation des forêts, dont témoignent l'abondance des taillis et surtout celle des chablis, l'appauvrissement des sols forestiers, et donc des écosystèmes, s'ils furent accentués par les hommes de la fin du Moyen Âge, étaient en marche depuis longtemps, quelquefois (comme pour les espaces de landes) depuis la protohistoire.

\section{Des héritages aux projets}

La question des héritages (et des projets) est au coeur des recherches actuelles, ce qui induit un état des milieux " hérités " dont l'action se fait sentir à des pas de temps différents. Cela a conduit à une remise en question de la périodisation classique, fondée en très large partie sur l'événementiel politique, à penser différemment les notions d'équilibre, de seuil et de rupture, de crise, ou encore de réversibilité ou d'irréversibilité des systèmes dont les sociétés actuelles héritent.

La réévaluation du passé et l'affirmation de la part des héritages dans les élaborations écouménales sont une affaire récente. Leur terrain d'élection aura été le champ de l'étude des milieux par la médiation de l'archéologie environnementale. L'installation de ce concept suppose l'abandon de deux notions étroitement liées. La première est l'idée de fixité des situations, la seconde est celle de dégradation des situations d'origine. L'une et l'autre produisent un récit linéaire, mécanique, où les enchaînements sont déduits des situations précédentes. Or, la question des héritages ne peut être réduite à cela. Les recherches sur la Loire en ont tracé les étapes et ont permis de poser la question de la dynamique en lieu et place de celle de la 
dégradation. C'est le sens qu'il faut donner à la succession des colloques d'Antibes. En 1995, en plein essor du programme Archaeomedes (financé par le programme Environnement de la DG XII de l'Union européenne), l'accent était mis sur le rôle de l'homme dans la dégradation de l'environnement. Dans la publication de 1997, c'était la dynamique qui était placée au premier plan. Il s'agissait de revisiter les concepts de nature, d'environnement et de paysage tels que les emploient les différentes corporations d'historiens, de développer un thème d'étude sur les interactions sociétés/ milieux, qui fasse la part des hybridations, des héritages et des mobilités. Ce thème a abouti à des formulations majeures telles que celle d'anthroposystème, celle de la mobilité de la nature à travers l'idée que les fleuves ont (aussi) une histoire, celle d'histoire des anthroposystèmes hérités développée au colloque d'Aix-en-Provence en 2002.

Au total, ce que change la notion d'héritage est fondamental. Elle installe une dynamique d'un autre type que la dynamique historique à coup d'intentionnalités sociales unilatérales, c'est-à-dire l'histoire comme récit des dégradations ou des planifications successives, sans interactions et sans récursivité. Elle insiste sur la part de mémoire dans la formation des hybrides. Elle débouche sur une théorie des scénarios (dite aussi histoire contrefactuelle) qu'il faut comprendre non pas comme le pouvoir de prévoir l'avenir, ce qui est une utopie, mais comme le fait de dégager les différentes histoires possibles, d'en voir une retenue par les chercheurs en fonction de l'état de l'art, tout en laissant la possibilité de réexaminer l'ensemble ultérieurement. Elle instaure une forme de contingence historique généralisée, y compris aux outils et aux concepts, et révèle des modèles historiques de gestion des ressources des milieux susceptibles d'offrir matière utile à la compréhension du fonctionnement contemporain de ces milieux, et de conduire à une implication plus lisible de l'historien (et du médiéviste en particulier) dans les questions de société en réponse à la demande sociale. À l'heure où il est beaucoup question de dégradation, de perte, de conservation et de patrimonialisation du vivant, réintroduire la notion du temps historique paraît particulièrement opportun, notamment lorsqu'on touche à toutes les questions concernant le problème de la biodiversité. Il faut éviter l'appropriation de l'histoire par des non-historiens. Ne pas laisser croire que l'on peut s'improviser historien, sous prétexte que le travail sur nos sources (écrites ou archéologiques) ne conduit pas à l'énoncé d'une formule chimique ou d'une équation mathématique pour laquelle un apprentissage est nécessaire. Ces acquis, croisés en permanence avec les analyses spatiales, introduisent à une réflexion prospective sur l'aménagement du territoire dans une confrontation permanente entre 
les « héritages » et les projets : dans cette dialectique, l'archéologie s'inscrit au cœur du débat entre les dynamiques sociales à l'œuvre dans le passé et les aménagements pour l'avenir. Ce dont nous souhaitons convaincre non seulement les spécialistes des documents écrits, mais aussi ceux des documents archéologiques, est que ces documentations doivent être, comme les autres, discutées de manière "interdisciplinaire " et que la pratique de l'interdisciplinarité conduit à poser les questions autrement, à connaître les limites de chaque type de document (échelle de sincérité d'Hélène Noizet, échelle de fiabilité de Joëlle Burnouf), à définir de nouvelles " procédures " d'analyse et de discussion des documents, et, partant, à définir de nouveaux " protocoles " et donc à collecter les données différemment.

Joëlle Burnouf

Université Paris 1 Panthéon-Sorbonne ArScAn (CNRS, UMR 7041), Nanterre

Corinne BECK

Université de Valenciennes et du Hainaut-Cambrésis ArScAn (CNRS, UMR 7041), Nanterre

Marie-Christine Bailly-Maître Centre national de la recherche scientifique LAMM (UMR 6572), Aix-en-Provence

François Ducepre-Lamarre Lycée Carnot, Bruay-la-Buissière IRHiS (CNRS, UMR 8529), Lille

Fabrice GuIZARD-DUChamp

Université de Valenciennes et du Hainaut-Cambrésis CRHICC (EA 2244)

Aline Durand

Université de Provence Aix-Marseille 1 LAMM (CNRS, UMR 6572), Aix-en-Provence

Carole Puig

SARL ACTER

FRAMESPA (CNRS, UMR 5136), Toulouse 


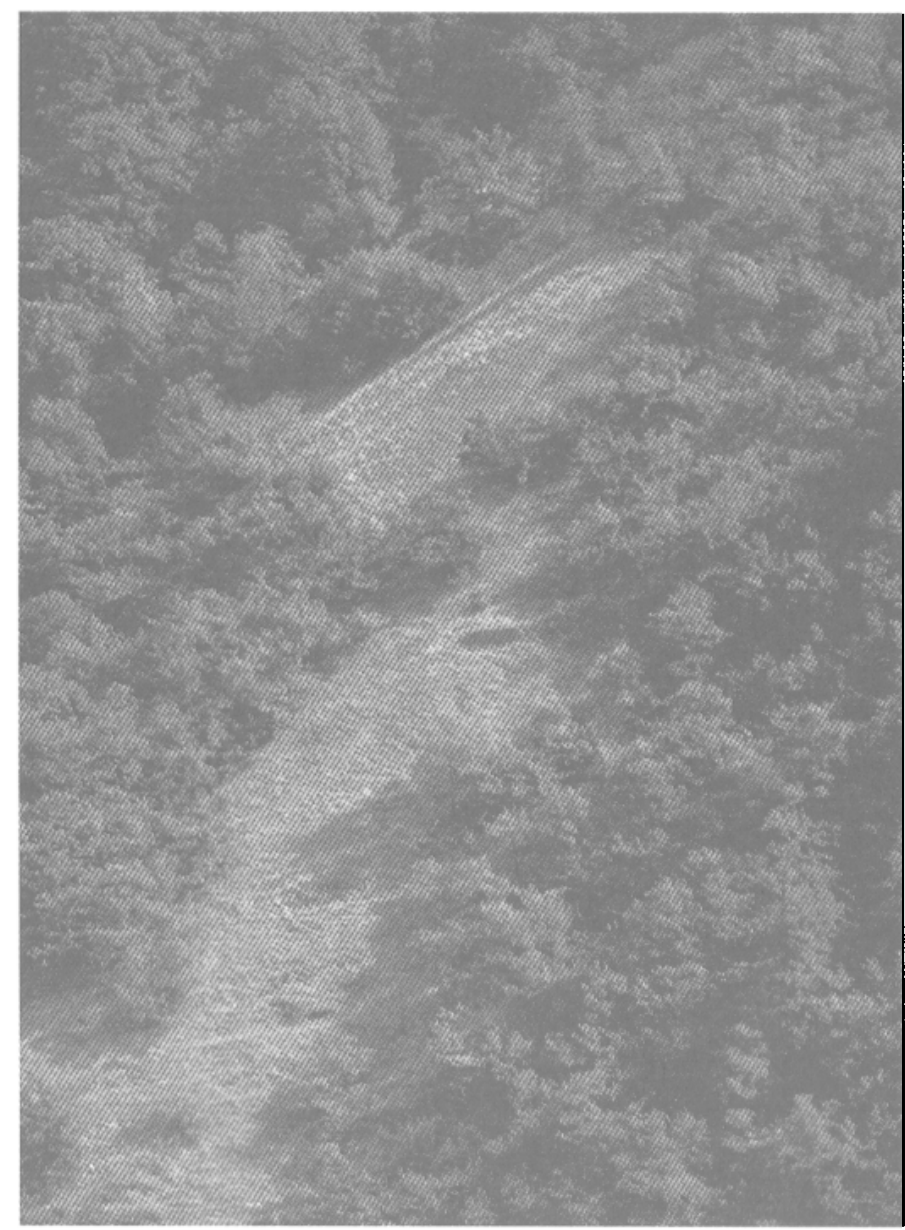

Figure 1. - Site de Petra Alba à Saint-Laurent-le-Minier (Gard) Les haldes formées il y a 800 ans marquent encore le paysage (cliché M.-Chr. Bailly-Maître).

Figure 2. - Site de Jarrier (Savoie) L'exploitation des failles minéralisées laisse des signes indélébiles dans l'environnement (cliché M.-Chr. Bailly-Maître).

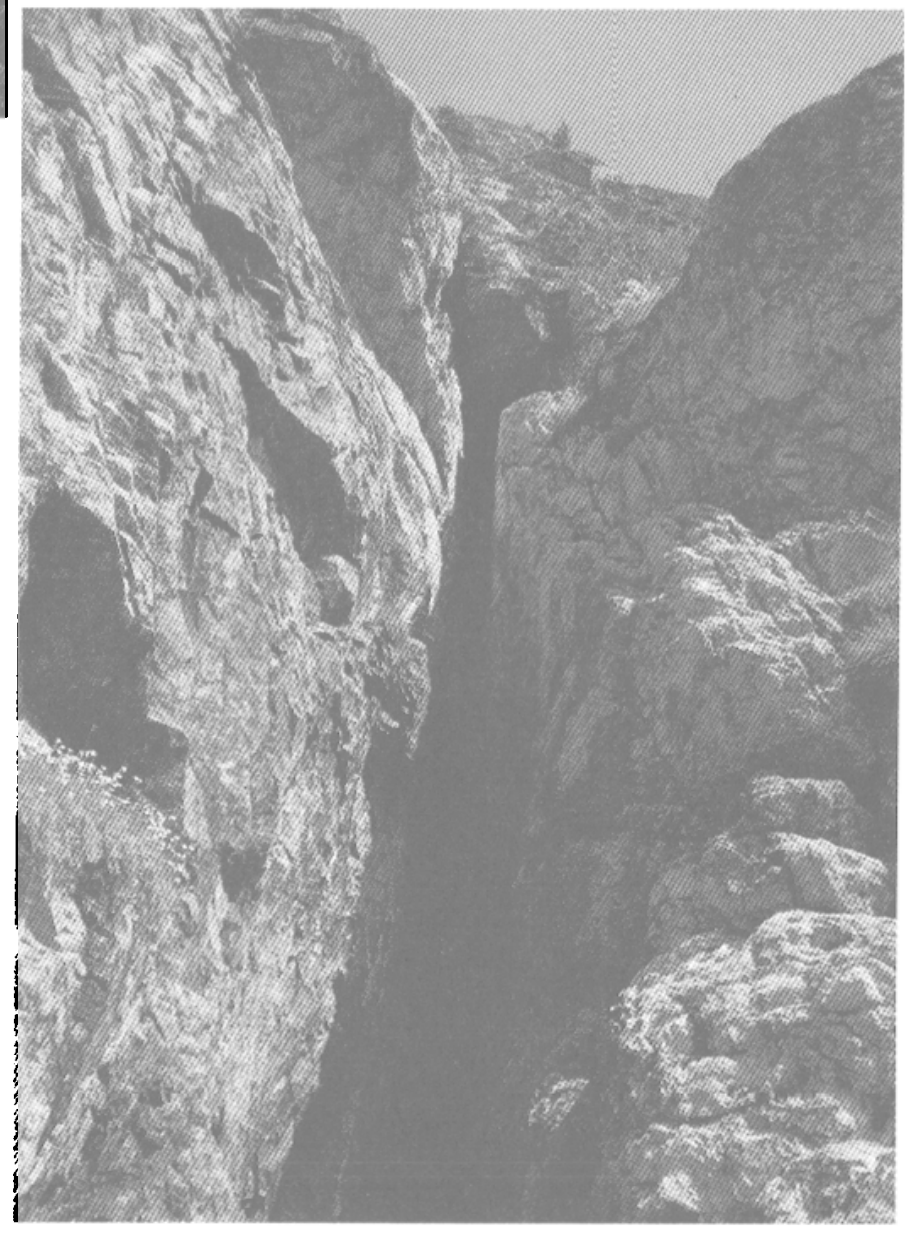


Sociétés, milieux, ressources : un nouveau paradigme

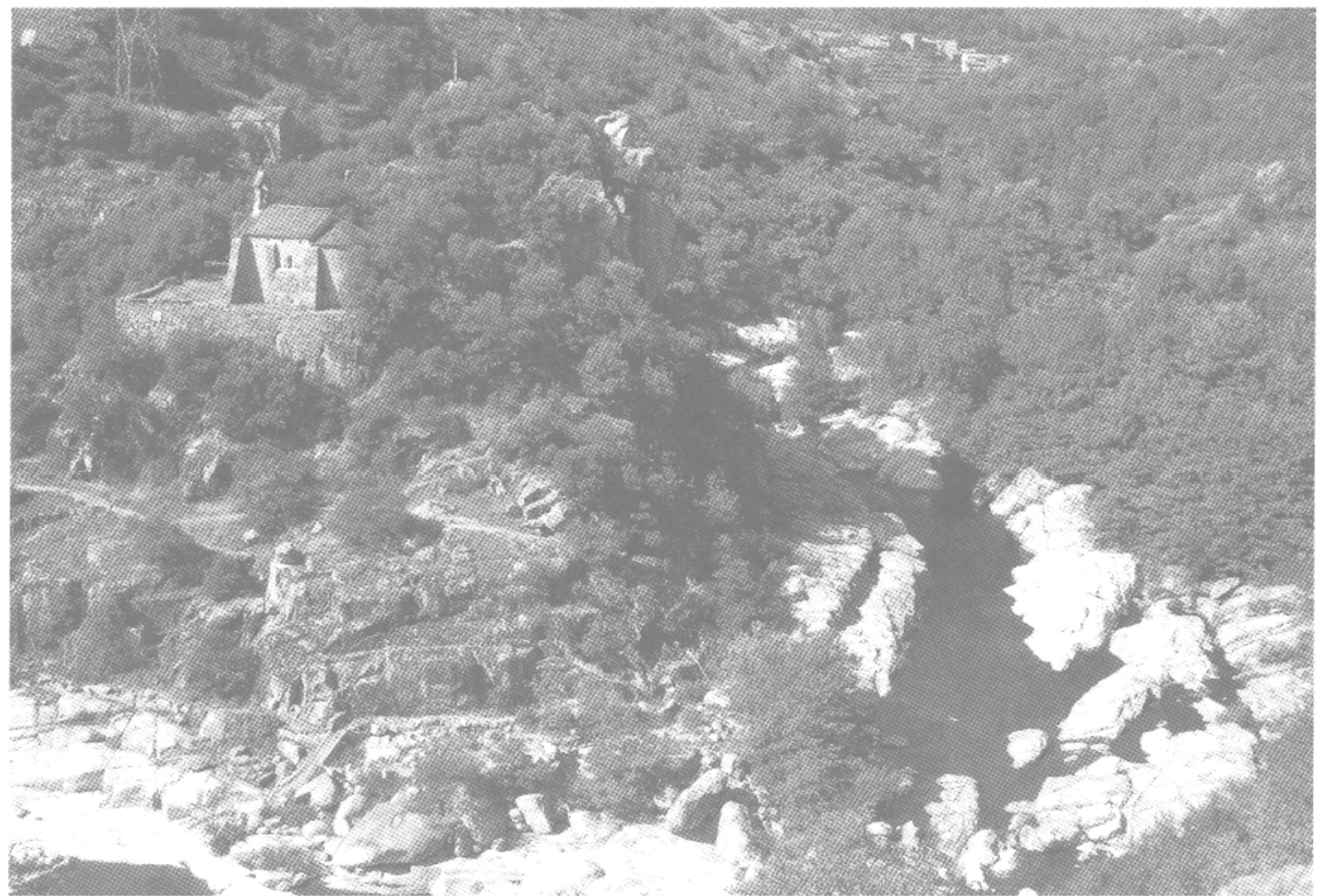

Figure 3. - Château et chapelle castrale de Pied-de-Borne (Lozère)

Cet ensemble fait partie du dispositif de surveillance du territoire minier de la vallée du Chassezac (cliché M.-Chr. Bailly-Maître).

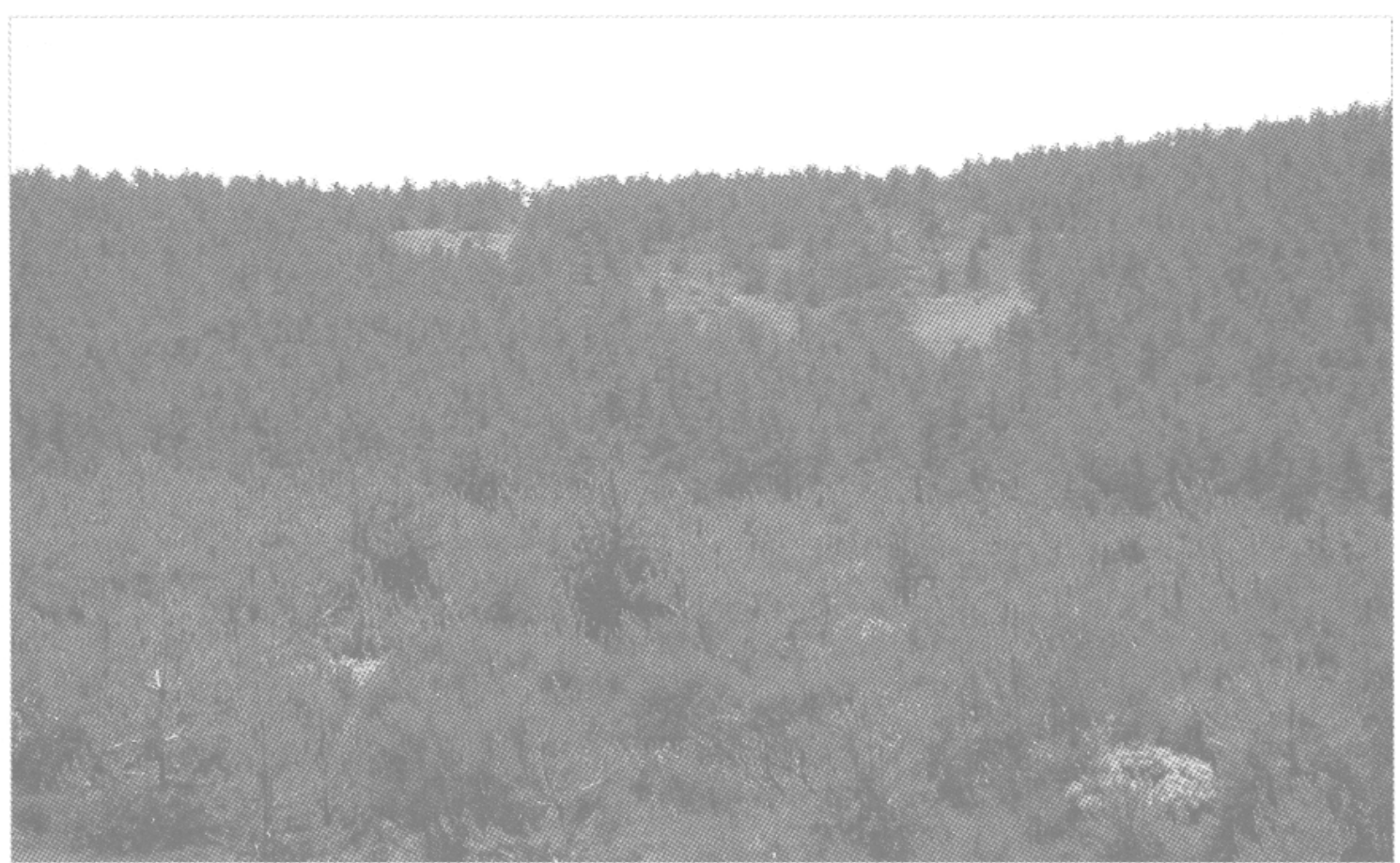

Figure 4. - Mont Lozère (Lozère)

La pollution engendrée par la métallurgie du plomb au XI ${ }^{\mathrm{c}}$ siècle se lit encore aujourd'hui dans le paysage (cliché M.-Chr. Bailly-Maître). 


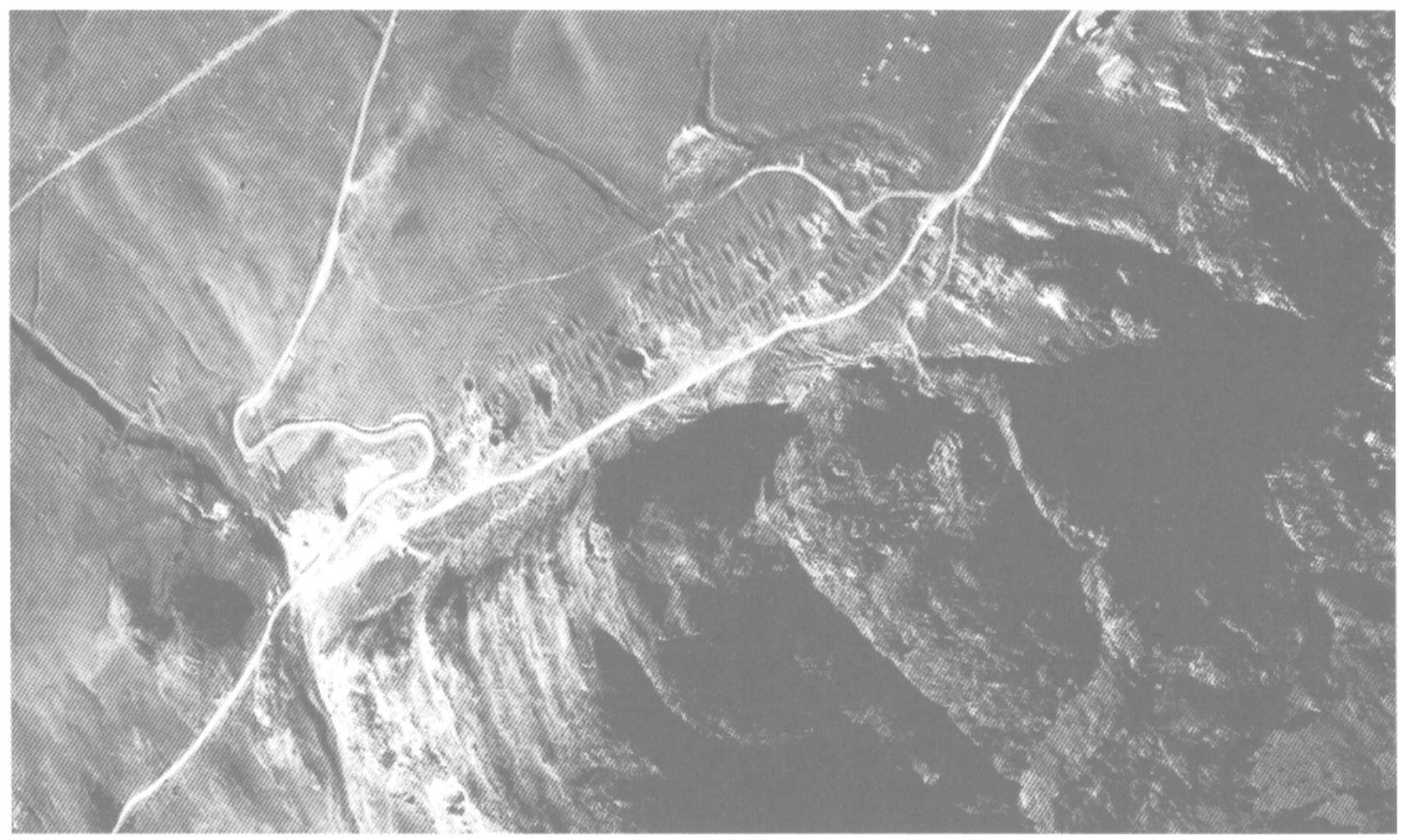

Figure 5. - Brandes-en-Oisans à Huez (Isère) La présence du minerai argentifere a provoqué la création de petites villes abandonnées dès l'arrêt de l'exploitation (cliché M.-Chr. Bailly-Maître).

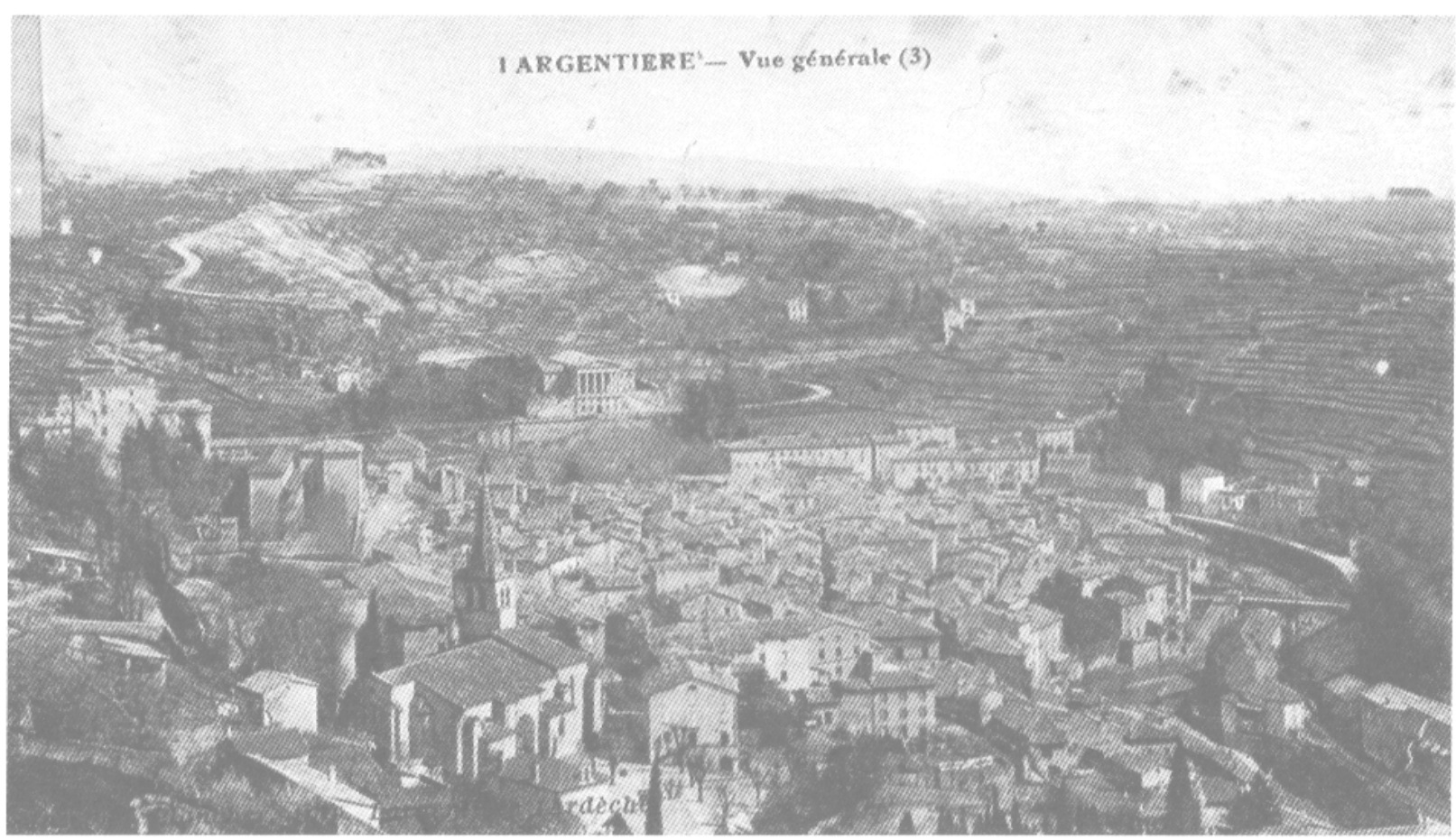

Figure 6. - Largentière (Ardèche)

La présence du minerai argentifere a provoqué la création de petites villes dont la survie, jusqu'à nos jours, est liée à la poursuite de l'exploitation (cliché M.-Chr. Bailly-Maître). 
Figure 7. - Arbres complantés dans une vigne,

Barthélemy l'Anglais, Livre des propriétés des choses, Paris, BnF, Fr. 9140, fol. 185v (France, 1470-1500) (C BnF. 
Figure 8. - Plantation d'arbres et de vigne dans la région de Perpignan (avec l'autorisation de l'auteur, Jérôme Kotarba. Extrait de J. Kotarba, A. Bergeret, D. Peyric et C. PuIG, RN9, Contournement du Grand Saint-Charles, Perpignan, Orle-Ouest agraire, DFS, SRA Languedoc-Roussillon, Montpellier, 2000). 